TRANSACTIONS OF THE

AMERICAN MATHEMATICAL SOCIETY

Volume 358, Number 6, Pages 2759-2779

S 0002-9947(06)03864-5

Article electronically published on January 27, 2006

\title{
BLOCK COMBINATORICS
}

\author{
V. FARMAKI AND S. NEGREPONTIS
}

\begin{abstract}
In this paper we extend the block combinatorics partition theorems of Hindman and Milliken-Taylor in the setting of the recursive system of the block Schreier families $\left(\mathcal{B}^{\xi}\right)$, consisting of families defined for every countable ordinal $\xi$. Results contain (a) a block partition Ramsey theorem for every countable ordinal $\xi$ (Hindman's Theorem corresponding to $\xi=1$, and the Milliken-Taylor Theorem to $\xi$ a finite ordinal), (b) a countable ordinal form of the block Nash-Williams partition theorem, and (c) a countable ordinal block partition theorem for sets closed in the infinite block analogue of Ellentuck's topology.
\end{abstract}

\section{INTRODUCTION}

In this paper we extend the block combinatorics partition theorems of Hindman [H] and Milliken-Taylor [M, T2 to countable ordinals in the setting of the recursive system of the block Schreier families $\mathcal{B}^{\xi}$ for $\xi<\omega_{1}$ (Definition 2.1). The main results are contained in Theorem 2.6. Theorem 4.4, Theorem 4.6 and Theorem 5.2.

Theorem 2.6 is a block Ramsey partition theorem for every countable ordinal $\xi$; the basic, starting dichotomy, corresponding to the ordinal level $\xi=1$ is Hindman's Theorem $[\mathrm{H}]$. We recall this theorem in the equivalent form proved (in a simpler and more elegant way) by Baumgartner in $[\mathrm{B}$. We denote by $\mathbb{N}$ the set $\{1,2, \ldots\}$, of all natural numbers and by $[\mathbb{N}]_{>0}^{<\omega}$ the set of all the finite nonempty subsets of $\mathbb{N}$.

Theorem 1.1 (Hindman's theorem, $[\mathrm{H}],[\mathrm{B}]$ ). Let $\mathcal{F} \subseteq[\mathbb{N}]_{>0}^{<\omega}$. Then, there exists an infinite subset $\mathcal{D}$ of $[\mathbb{N}]_{>0}^{<\omega}$ such that all its elements are pairwise disjoint, and if $F U(\mathcal{D})$ is the set of all finite unions of members of $\mathcal{D}$, then

either $F U(\mathcal{D}) \subseteq \mathcal{F}$ or $F U(\mathcal{D}) \subseteq[\mathbb{N}]_{>0}^{<\omega} \backslash \mathcal{F}$.

Hindman's Theorem has been extended independently by Milliken [M, Theorem 2.2], in sumset form, and Taylor [T2, Lemma 2.2], in Baumgartner's formulation; for its statement we introduce some notation. For $s, t \in[\mathbb{N}]_{>0}^{<\omega}$ we write $s<t$ if $\max s<\min t$. For $k \in \mathbb{N}$

$$
\begin{aligned}
& \mathcal{B}^{k}=\left\{\mathbf{s}=\left(s_{1}, \ldots, s_{k}\right): s_{1}<\cdots<s_{k} \in[\mathbb{N}]_{>0}^{<\omega}\right\} ; \text { and } \\
& \mathcal{B}^{\omega}(\mathbb{N})=\left\{\mathcal{D}=\left(s_{n}\right)_{n \in \mathbb{N}}: s_{n} \in[\mathbb{N}]_{>0}^{<\omega} \text { and } s_{n}<s_{n+1} \forall n \in \mathbb{N}\right\} .
\end{aligned}
$$

For $\mathcal{D}=\left(t_{n}\right)_{n \in \mathbb{N}} \in \mathcal{B}^{\omega}(\mathbb{N})$, let $F U(\mathcal{D})=\left\{\bigcup_{n \in H} t_{n}: H \in[\mathbb{N}]_{>0}^{<\omega}\right\}$, and

$$
\mathcal{B}^{<\omega}(\mathcal{D})=\left\{\mathbf{s}=\left(s_{1}, \ldots, s_{k}\right): k \in \mathbb{N}, s_{1}<\cdots<s_{k} \in F U(\mathcal{D})\right\} \cup\{\emptyset\} \text {. }
$$

Received by the editors June 9, 2004 and, in revised form, September 9, 2004.

2000 Mathematics Subject Classification. Primary 03E05; Secondary 05D10, 46B20.

Key words and phrases. Block Ramsey, Nash-Williams combinatorics, Schreier families.

(C)2006 American Mathematical Society Reverts to public domain 28 years from publication 
Theorem 1.2 (Milliken-Taylor theorem, $\mathrm{M}$, T2]). Let $k \in \mathbb{N}, \mathcal{F} \subseteq \mathcal{B}^{k}$ and $\mathcal{D}_{0} \in \mathcal{B}^{\omega}(\mathbb{N})$. Then there exists $\mathcal{D}=\left(s_{n}\right)_{n \in \mathbb{N}} \in \mathcal{B}^{\omega}(\mathbb{N})$ with $s_{n} \in F U(\mathcal{D})$ for every $n \in \mathbb{N}$ such that

$$
\text { either } \mathcal{B}^{k} \cap \mathcal{B}^{<\omega}(\mathcal{D}) \subseteq \mathcal{F} \text { or } \mathcal{B}^{k} \cap \mathcal{B}^{<\omega}(\mathcal{D}) \subseteq \mathcal{B}^{k} \backslash \mathcal{F}
$$

It is clear that the Milliken-Taylor theorem is analogous to the classical Ramsey partition theorem, where "singletons" in $\mathbb{N}$ are replaced by "finite sets" in $\mathbb{N}$. Now, Ramsey's partition theorem has been extended (in F2, F3) to a partition theorem holding for every countable ordinal number, in a way that the classical theorem corresponds to the case of finite ordinals. Instrumental for this extension is the definition of the recursive system of thin Schreier families $\left(\mathcal{A}_{\xi}\right)_{\xi<\omega_{1}}$, given below, where (in case (3iii)) the Cantor normal form of ordinals (cf. [KM], $[\mathrm{L}$ ) is employed:

Definition 1.3 (The recursive thin Schreier system, [F1, Def. 7], [F2, Def. 1.5], [F3, Def. 1.3]). For every nonzero countable limit ordinal $\lambda$ choose and fix a strictly increasing sequence $\left(\lambda_{n}\right)$ of successor ordinals smaller than $\lambda$ with $\sup \lambda_{n}=\lambda$. The system $\left(\mathcal{A}_{\xi}\right)_{\xi<\omega_{1}}$ is defined recursively as follows:

(1) $\mathcal{A}_{0}=\{\emptyset\}$ and $\mathcal{A}_{1}=\{\{n\}: n \in \mathbb{N}\}$;

(2) for $\zeta>0, \mathcal{A}_{\zeta+1}=\left\{s \in[\mathbb{N}]_{>0}^{<\omega}: s=\{n\} \cup s_{1}\right.$, where $n \in \mathbb{N},\{n\}<s_{1}$ and $\left.s_{1} \in \mathcal{A}_{\zeta}\right\}$

(3i) $\mathcal{A}_{\omega^{\beta+1}}=\left\{s \in[\mathbb{N}]_{>0}^{<\omega}: s=\bigcup_{i=1}^{n} s_{i}\right.$, where $n=\min s_{1}, s_{1}<\cdots<s_{n}$ and $\left.s_{1}, \ldots, s_{n} \in \mathcal{A}_{\omega^{\beta}}\right\}$

(3ii) for a nonzero, countable limit ordinal $\lambda$, $\mathcal{A}_{\omega^{\lambda}}=\left\{s \in[\mathbb{N}]_{>0}^{<\omega}: s \in \mathcal{A}_{\omega^{\lambda_{n}}}\right.$ with $\left.n=\min s\right\} ;$ and

(3iii) for a limit ordinal $\xi$ such that $\omega^{a}<\xi<\omega^{a+1}$ for some $0<a<\omega_{1}$, if $\xi=\omega^{a} p+\sum_{i=1}^{m} \omega^{a_{i}} p_{i}$, where $m \in \mathbb{N}$ with $m \geq 0, p, p_{1}, \ldots, p_{m}$ are natural numbers with $p, p_{1}, \ldots, p_{m} \geq 1$ (so that either $p>1$, or $p=1$ and $m \geq 1$ ) and $a, a_{1}, \ldots, a_{m}$ are ordinals with $a>a_{1}>\cdots a_{m}>0$, $\mathcal{A}_{\xi}=\left\{s \in[\mathbb{N}]_{>0}^{<\omega}: s=s_{0} \cup\left(\bigcup_{i=1}^{m} s_{i}\right)\right.$ with $s_{m}<\cdots<s_{1}<s_{0}, s_{0}=$ $s_{1}^{0} \cup \cdots \cup s_{p}^{0}$ with $s_{1}^{0}<\cdots<s_{p}^{0} \in \mathcal{A}_{\omega^{a}}$, and $s_{i}=s_{1}^{i} \cup \cdots \cup s_{p_{i}}^{i}$ with $\left.s_{1}^{i}<\cdots<s_{p_{i}}^{i} \in \mathcal{A}_{\omega^{a_{i}}} \forall 1 \leq i \leq m\right\}$.

It will be remarked that each family $\mathcal{A}_{\xi}$ consists of finite subsets of $\mathbb{N}$, that $\mathcal{A}_{k}=[\mathbb{N}]^{k}$ for every $k \in \mathbb{N}$, and that $\mathcal{A}_{\xi}$ contains finite sets of arbitrarily large cardinality for every ordinal $\xi \geq \omega$. The families $\mathcal{A}_{\omega}=\left\{s \in[\mathbb{N}]_{>0}^{<\omega}: s \in[\mathbb{N}]^{k}\right.$, where $k=\min s\}$ and $\mathcal{A}_{\omega^{n+1}}=\left\{s \in[\mathbb{N}]_{>0}^{<\omega}: s=\bigcup_{i=1}^{k} s_{i}\right.$, where $k=\min s_{1}$, $\left.s_{1}<\cdots<s_{k} \in \mathcal{A}_{\omega^{n}}\right\}$ are determined by case (3i) of Definition 1.3. In general however the Schreier families are not defined in a unique way, but depend ultimately on the choice of the converging sequence $\left(\lambda_{n}\right)$ to the limit ordinals $\lambda$. This choice bears directly on case (3ii) and indirectly on all other cases of Definition 1.3. The first real choice is for the limit ordinal $\lambda=\omega$ and, assuming that the sequence $\left(\lambda_{n}\right)$ converging to $\omega$ is the sequence with $\lambda_{n}=n, \mathcal{A}_{\omega^{\omega}}=\left\{s \in[\mathbb{N}]_{>0}^{<\omega}: s \in \mathcal{A}_{\omega^{k}}\right.$ with $k=\min s\}$. By choosing "natural" converging sequences $\lambda_{n}=\omega+n$ to limit ordinal $\lambda=\omega 2, \ldots, \lambda_{n}=\omega^{n}+1$ to limit ordinal $\lambda=\omega^{\omega}$, the families $\mathcal{A}_{\omega^{\omega 2}}, \ldots, \mathcal{A}_{\omega^{\omega} \omega}$ are defined, respectively. More "natural" converging sequences are considered in Section 2 of [KS] (up to the ordinal $\epsilon_{0}$ ), and in Chapter VII of [KM]. However, it appears impossible to make canonical, natural choices for all limit ordinals, and it thus seems that the definition of the recursive system of thin Schreier families $\left(\mathcal{A}_{\xi}\right)_{\xi<\omega_{1}}$ depends essentially on the arbitrary choices of (many) converging sequences. 
It must be emphasised however that our results (in Sections 2-5) do not depend on the particular choice of the converging sequences. The basic feature that differentiates the $\mathcal{A}_{\xi}$ 's from each other is complexity: in fact, irrespective of the particular choices of the converging sequences employed for the definition of the family $\mathcal{A}_{\xi}$, the Cantor-Bendixson index of the compact subset (in the Cantor set $\{0,1\}^{\mathbb{N}}$, under the canonical identification of a set with its characteristic function) corresponding to $\mathcal{A}_{\xi}$ is precisely $\xi+1$ (as proved in [F3, Proposition 2.9]). Thus the complexity of the family $\mathcal{A}_{\xi}$, as measured by its Cantor-Bendixson index, is independent of the particular choices of the converging sequences. This invariance is in fact needed for the results in Sections 4 and 5 below.

Although the recursive system of Schreier families $\left(\mathcal{A}_{\xi}\right)$ is a purely combinatorial entity, intimately related, as it turns out, with Ramsey and Nash-Williams combinatorics, it nevertheless arose gradually in connection with the theory of Banach spaces, originally by Schreier $[\underline{\mathrm{S}}$, (for $\xi=\omega$ ), next by Alspach-Odell [AO] (for $\xi=\omega^{\kappa}, \kappa$ a natural number) and Alspach-Argyros [AA] (for $\xi=\omega^{\alpha}, \alpha$ a countable ordinal), and finally by Farmaki [F1], [F2], F3] and Tomczak-Jaegermann [TJ] (for $\xi$ any countable ordinal). (The reader is referred to the introduction of [F3] for more details.) In fact, variants of the Schreier families have found essential applications in Banach space theory on such questions as unconditionality, $l^{1}$ and $c_{0}$ embeddability, and distortion (see e.g. F1, O], AGR, F4]).

It is also noteworthy that the hereditary family $\left(\mathcal{A}_{\omega}\right)_{*}=\left\{t \in[\mathbb{N}]^{<\omega}: t \subseteq s\right.$ for some $\left.s \in \mathcal{A}_{\omega}\right\} \cup\{\emptyset\}$ generated by $\mathcal{A}_{\omega}$ figures prominently (under the name of the family of "not large" sets) in fundamental questions of mathematical logic, specifically in the (Ramsey type) Paris-Harrington statements, statements true and provable in set-theory but unprovable in Peano arithmetic (cf. [PH] and [KS]). The higher order hereditary Schreier families $\left(\mathcal{A}_{\xi}\right)_{*}$ might well be useful in forming and proving statements true but unprovable in certain systems endowed with induction stronger than that in Peano arithmetic.

In the present paper, we initially introduce (in Definition 2.1 below) the "block" analogue $\left(\mathcal{B}^{\xi}\right)_{\xi<\omega_{1}}$ of the recursive thin Schreier system $\left(\mathcal{A}_{\xi}\right)_{\xi<\omega_{1}}$. With its help we obtain (in Theorem 2.6 below) a suitable ordinal extension of the Hindman, Milliken-Taylor partition theorems for every countable ordinal.

Next we turn our attention to Nash-Williams type partition theorems. Denoting by $[L]$ the family of all the infinite subsets of a set $L$, our starting point is the classical

Theorem 1.4 (Nash-Williams theorem, $[\mathrm{NW}]$ ). Let $\mathcal{U}$ be a subfamily of $[\mathbb{N}]$, closed in the relative topology of $[\mathbb{N}]$, considered as a subspace of the Cantor set $\{0,1\}^{\mathbb{N}}$ with the product topology, and $M \in[\mathbb{N}]$. Then, there exists $L \in[M]$ such that

$$
\text { either }[L] \subseteq \mathcal{U} \text { or }[L] \subseteq[\mathbb{N}] \backslash \mathcal{U}
$$

An analogue of this classical theorem for blocks (stated as Corollary 4.10 below) has been established by Milliken [M, Theorem 3.5] and independently by Taylor [T1].

Using Theorem 2.6, together with some additional properties of the block Schreier families $\mathcal{B}^{\xi}$ for $\xi<\omega_{1}$ (proved in Section 3 below), including the computation of their Cantor-Bendixson index (Proposition 3.11) and the canonical representation of every, finite or infinite, ordered disjoint collection with respect to every family $\mathcal{B}^{\xi}$ 
(Proposition 3.5), we obtain in Theorem 4.4 Theorem 4.6 and Corollary 4.8 strong countable ordinal forms of the block Nash-Williams partition theorem. Finally, in Section 5, block Ellentuck type partition theorems are considered.

The combinatorial nature of the block Schreier families $\mathcal{B}^{\xi}$ for $\xi<\omega_{1}$ and of the block combinatorics developed in this paper makes it reasonable to expect that the results presented in this paper will find substantial applications in Ramsey theory proper (as it is expounded in GRS ) and in various other branches of mathematics.

We will need the following notations.

Some additional notation. For a set $X$ and a natural number $n$ we denote by $[X]^{n}$ the set of all the $n$-element subsets of $X$, by $[X]^{<\omega}$ the set of all the finite subsets of $X$, by $[X]_{>0}^{<\omega}$ the set of all the finite nonempty subsets of $X$, and by $[X]$ the set of all the infinite subsets of $X$. If $s \in[\mathbb{N}]^{<\omega}$ and $t \subseteq \mathbb{N}$ we write $s<t$ in place of "for all $x \in s$ and $y \in t, x<y$ ", while $s \propto t$ if $s$ is an initial segment of $t$, which means that there exists $r \subseteq \mathbb{N}$ with $s<r$ and $s \cup r=t$.

An ordered disjoint collection $D$ is a (finite or infinite) subset of $[\mathbb{N}]_{>0}^{<\omega}$ with the property that either $s<t$ or $t<s$ whenever $s$ and $t$ are distinct elements of $D$. We define the set $\mathcal{B}^{<\omega}(\mathbb{N})$ of all finite-ordered disjoint collections, considering them as strictly increasing finite sequences and analogously the set $\mathcal{B}^{\omega}(\mathbb{N})$ of all infinite-ordered disjoint collections as follows:

$\mathcal{B}^{<\omega}(\mathbb{N})=\left\{\mathbf{s}=\left(s_{1}, \ldots, s_{k}\right): k \in \mathbb{N}, s_{1}<\cdots<s_{k} \in[\mathbb{N}]_{>0}^{<\omega}\right\} \cup\{\emptyset\} ;$

$\mathcal{B}^{\omega}(\mathbb{N})=\left\{\mathcal{D}=\left(s_{n}\right)_{n \in \mathbb{N}}: s_{n} \in[\mathbb{N}]_{>0}^{<\omega}\right.$ and $\left.s_{n}<s_{n+1} \forall n \in \mathbb{N}\right\}$.

Let $\mathbf{s}=\left(s_{1}, \ldots, s_{k}\right) \in \mathcal{B}^{<\omega}(\mathbb{N})$ and $\mathcal{D}=\left(t_{n}\right)_{n \in \mathbb{N}} \in \mathcal{B}^{\omega}(\mathbb{N})$. We set

$F U(\mathcal{D})=\left\{\bigcup_{n \in H} t_{n}: H \in[\mathbb{N}]_{>0}^{<\omega}\right\}$ and $F U(\mathbf{s})=\left\{\bigcup_{n \in H} s_{n}: \emptyset \neq H \subseteq\right.$ $\{1, \ldots, k\}\}$.

For $\mathcal{D}_{1}, \mathcal{D}_{2} \in \mathcal{B}^{\omega}(\mathbb{N})$ we write $\mathcal{D}_{1}<\mathcal{D}_{2}$ if $\mathcal{D}_{1}$ is a sequence in $F U\left(\mathcal{D}_{2}\right)$. We set

$\mathcal{B}^{<\omega}(\mathcal{D})=\left\{\mathbf{s}=\left(s_{1}, \ldots, s_{k}\right) \in \mathcal{B}^{<\omega}(\mathbb{N}): k \in \mathbb{N}, s_{1}<\cdots<s_{k} \in F U(\mathcal{D})\right\} \cup\{\emptyset\} ;$

$\mathcal{B}^{\omega}(\mathcal{D})=\left\{\mathcal{D}_{1} \in \mathcal{B}^{\omega}(\mathbb{N}): \mathcal{D}_{1}<\mathcal{D}\right\}$.

Let $\mathbf{s}=\left(s_{1}, \ldots, s_{k}\right), \mathbf{t}=\left(t_{1}, \ldots, t_{m}\right) \in \mathcal{B}^{<\omega}(\mathbb{N})$ and $\mathcal{D}=\left(t_{n}\right)_{n \in \mathbb{N}} \in \mathcal{B}^{\omega}(\mathbb{N})$. We say that $\mathbf{s}$ is an initial segment of $\mathcal{D}$ (resp. $\mathbf{s}$ is an initial segment of $\mathbf{t}$ ), and we write $\mathbf{s} \propto \mathcal{D}($ resp. $\mathbf{s} \propto \mathbf{t})$ if $s_{i}=t_{i}$ for every $i=1, \ldots, k$. Let $t \in[\mathbb{N}]_{>0}^{<\omega}$ and $l=\min \left\{n \in \mathbb{N}: t<t_{n}\right\}$; then we set

$\mathcal{D}-t=\left(t_{l}, t_{l+1}, \ldots\right)$ and $\mathcal{D}-\mathbf{s}=\mathcal{D}-s_{k}$.

For a family $\mathcal{F} \subseteq \mathcal{B}^{<\omega}(\mathbb{N})$ and $t \in[\mathbb{N}]_{>0}^{<\omega}$ we set

$\mathcal{F}(t)=\left\{\mathbf{s} \in \mathcal{B}^{<\omega}(\mathbb{N}): \mathbf{s}=\left(s_{1}, \ldots, s_{k}\right)\right.$ and $\left(t, s_{1}, \ldots, s_{k}\right) \in \mathcal{F}$ or $\mathbf{s}=\emptyset$ and

$(t) \in \mathcal{F}\}$.

$\mathcal{F}-t=\left\{\mathbf{s}=\left(s_{1}, \ldots, s_{k}\right) \in \mathcal{F}: t<s_{1}<\cdots<s_{k}\right\}$.

For a family $\mathcal{A}$ of finite subsets of $\mathbb{N}$ and $n \in \mathbb{N}$ we set

$\mathcal{A}(n)=\left\{s \in[\mathbb{N}]^{<\omega}:\{n\}<s\right.$ and $\left.\{n\} \cup s \in \mathcal{A}\right\}$.

\section{The Block RAMSEy PARTITION TheOREM FOR EVERY COUNTABle ORDinAL}

The purpose of this section is to prove the block Ramsey partition theorem for every countable ordinal (Theorem 2.6). It is the extension to every countable ordinal $\xi$ of Hindman's theorem (Theorem 1.1) corresponding to ordinal level $\xi=1$ and Milliken-Taylor theorem (Theorem 1.2), obtained independently by Milliken [M, Theorem 2.2] and Taylor [T2, Lemma 2.2], corresponding to finite ordinals $\xi<\omega$.

The vehicle of proving this extended block Ramsey partition theorem is the recursive thin block Schreier system $\left(\mathcal{B}^{\xi}\right)_{\xi<\omega_{1}}$ (Definition 2.1), consisted of families 
of finite-ordered disjoint collections. This is similar to the recursive thin Schreier system $\left(\mathcal{A}_{\xi}\right)_{\xi<\omega_{1}}$ (Definition 1.3) of families of finite subsets of $\mathbb{N}$.

Definition 2.1 (Recursive thin block Schreier system $\left(\mathcal{B}^{\xi}\right)_{\xi<\omega_{1}}$ ). We define

$$
\mathcal{B}^{0}=\{\emptyset\}
$$

and for every countable ordinal $\xi \geq 1$

$$
\begin{gathered}
\mathcal{B}^{\xi}=\left\{\mathbf{s}=\left(s_{1}, \ldots, s_{k}\right): k \in \mathbb{N}, s_{1}<\cdots<s_{k} \in[\mathbb{N}]_{>0}^{<\omega}\right. \\
\text { and } \left.\left\{\min s_{1}, \ldots, \min s_{k}\right\} \in \mathcal{A}_{\xi}\right\} .
\end{gathered}
$$

Remark 2.2. $\quad$ (i) $\mathcal{B}^{\xi} \subseteq \mathcal{B}^{<\omega}(\mathbb{N})$ and $\emptyset \notin \mathcal{B}^{\xi}$ for $\xi \geq 1$.

(ii) For $k \in \mathbb{N}, \mathcal{B}^{k}=\left\{\left(s_{1}, \ldots, s_{k}\right): s_{1}<\cdots<s_{k} \in[\mathbb{N}]_{>0}^{<\omega}\right\}$,

$\mathcal{B}^{\omega}=\left\{\left(s_{1}, \ldots, s_{k}\right): k \in \mathbb{N}, s_{1}<\cdots<s_{k} \in[\mathbb{N}]_{>0}^{<\omega}\right.$ and $\left.\min s_{1}=k\right\}$,

$\mathcal{B}^{\omega^{n}}=\left\{\left(s_{1}, \ldots, s_{k}\right) \in B^{<\omega}(\mathbb{N})\right.$ : if $\min s_{1}=m$ there exist $k_{1}<\cdots<k_{m}=k \in$

$\mathbb{N}$ such that $\left.\left(s_{1}, \ldots, s_{k_{1}}\right), \ldots,\left(s_{k_{m-1}+1}, \ldots, s_{k_{m}}\right) \in \mathcal{B}^{\omega^{n-1}}\right\}$ for every $n \in \mathbb{N}$, and

$\mathcal{B}^{\omega^{\omega}}=\left\{\left(s_{1}, \ldots, s_{k}\right) \in B^{<\omega}(\mathbb{N}):\left(s_{1}<\cdots<s_{k}\right) \in \mathcal{B}^{\omega^{m}}\right.$ if $\left.\min s_{1}=m\right\}$, in case the cofinal sequence chosen and fixed for the limit ordinal $\lambda=\omega$ in the definition of the system $\left(\mathcal{A}_{\xi}\right)_{\xi<\omega_{1}}$ is $(n)_{n \in \mathbb{N}}$. One defines analogously the families $\mathcal{B}^{\omega^{\omega(p+1)}}$ for $p \in \mathbb{N}, \mathcal{B}^{\omega^{\omega^{k}}}$ for $k \in \mathbb{N}, \mathcal{B}^{\omega^{\omega \omega}}$ and so on in case the chosen cofinal sequences for the the limit ordinals $\omega(p+1), \omega^{k}, \omega^{\omega}$ are $(\omega p+n)_{n \in \mathbb{N}},\left(\omega^{k-1} n+1\right)_{n \in \mathbb{N}},\left(\omega^{n} n+1\right)_{n \in \mathbb{N}}$, respectively.

(iii) Of course the definition of the recursive thin block Schreier system $\left(\mathcal{B}^{\xi}\right)_{\xi<\omega_{1}}$ depends on the choice of the cofinal sequences $\left(\lambda_{n}\right)$ for each nonzero countable limit ordinal $\lambda$ in the definition of the corresponding Schreier system $\left(\mathcal{A}_{\xi}\right)_{\xi<\omega_{1}}$. However the fundamental combinatorial properties of the system, and in particular the complexity of the Schreier families of every ordinal index, as measured by the Cantor-Bendixson index (in Proposition 3.11 below), is, as remarked in the Introduction, independent of the particular choices and in fact invariant; in this sense the choice of the cofinal sequences is immaterial in the results of this paper.

The following proposition justifies the term "recursive" in our definition of the system $\left(\mathcal{B}^{\xi}\right)_{\xi<\omega_{1}}$.

Proposition 2.3. For every countable ordinal $\xi \geq 1$ there exists a concrete sequence $\left(\xi_{n}\right)$ of countable ordinals with $\xi_{n}<\xi$ such that for every $s \in[\mathbb{N}]_{>0}^{<\omega}$ with $\min s=n$

$$
\mathcal{B}^{\xi}(s)=\mathcal{B}^{\xi_{n}} \cap\left(\mathcal{B}^{<\omega}(\mathbb{N})-s\right) .
$$

Moreover, $\xi_{n}=\zeta$ for every $n \in \mathbb{N}$ if $\xi=\zeta+1$ and $\left(\xi_{n}\right)$ is a strictly increasing sequence with $\sup _{n} \xi_{n}=\xi$ if $\xi$ is a limit ordinal.

Proof. According to Proposition 1.7 in [F3, for every countable ordinal $\xi>0$ there exists a concrete sequence $\left(\xi_{n}\right)$ of countable ordinals with $\xi_{n}<\xi$ such that

$$
\mathcal{A}_{\xi}(n)=\mathcal{A}_{\xi_{n}} \cap[\{n+1, n+2, \ldots\}]^{<\omega} \text { for every } n \in \mathbb{N} .
$$

Moreover, $\xi_{n}=\zeta$ for every $n \in \mathbb{N}$ if $\xi=\zeta+1$ and $\left(\xi_{n}\right)$ is a strictly increasing sequence with $\sup _{n} \xi_{n}=\xi$ if $\xi$ is a limit ordinal

For $\xi=1$, we have $\mathcal{B}^{1}=\left\{\left(s_{1}\right): s_{1} \in[\mathbb{N}]_{>0}^{<\omega}\right\}$, hence, $\mathcal{B}^{1}(s)=\mathcal{B}^{0}=\{\emptyset\}$ for every $s \in[\mathbb{N}]_{>0}^{<\omega}$. Let $1<\xi<\omega_{1}$ and $s \in[\mathbb{N}]_{>0}^{<\omega}$ with $\min s=n$. If $\left(s_{1}, \ldots, s_{k}\right) \in \mathcal{B}^{\xi}(s)$, then $\left(s, s_{1}, \ldots, s_{k}\right) \in \mathcal{B}^{\xi}$ and $\left\{n, \min s_{1}, \ldots, \min s_{k}\right\} \in \mathcal{A}_{\xi}$. Hence, $\left\{\min s_{1}, \ldots, \min s_{k}\right\} \in \mathcal{A}_{\xi_{n}}$ and $\left(s_{1}, \ldots, s_{k}\right) \in \mathcal{B}^{\xi_{n}} \cap\left(\mathcal{B}^{<\omega}(\mathbb{N})-s\right)$. On 
the other hand, if $\left(s_{1}, \ldots, s_{k}\right) \in \mathcal{B}^{\xi_{n}} \cap\left(\mathcal{B}^{<\omega}(\mathbb{N})-s\right)$, then $\left\{\min s_{1}, \ldots, \min s_{k}\right\} \in$ $\mathcal{A}_{\xi_{n}} \cap[\{n+1, n+2, \ldots\}]^{<\omega} \subseteq \mathcal{A}_{\xi}(n)$. Hence, $\left\{n, \min s_{1}, \ldots, \min s_{k}\right\} \in \mathcal{A}_{\xi}$ and then $\left(s, s_{1}, \ldots, s_{k}\right) \in \mathcal{B}^{\xi}$.

In the classical Ramsey theory, the basic, starting dichotomy, corresponding to ordinal level $\xi=1$, is the statement that if we partition in two parts an infinite subset of $\mathbb{N}$, then at least one part is infinite. For the block Ramsey theory, that we are about to develop, the basic starting dichotomy, corresponding to ordinal level $\xi=1$, is (the highly nontrivial) Hindman's theorem (Theorem 1.1). For our purposes the following equivalent version of Hindman's theorem is more convenient.

Theorem 2.4 (Second version of Hindman's theorem, $[\underline{\mathrm{H}}, \underline{\mathrm{B}})$ ). Let $\mathcal{F} \subseteq \mathcal{B}^{<\omega}(\mathbb{N})$ be a family of finite-ordered disjoint collections and $\mathcal{D}_{0} \in \mathcal{B}^{\omega}(\mathbb{N})$ an infinite-ordered disjoint collection. Then, there exists an infinite-ordered disjoint collection $\mathcal{D}<\mathcal{D}_{0}$ such that:

$$
\text { either } \mathcal{B}^{1} \cap \mathcal{B}^{<\omega}(\mathcal{D}) \subseteq \mathcal{F} \text { or } \mathcal{B}^{1} \cap \mathcal{B}^{<\omega}(\mathcal{D}) \subseteq \mathcal{B}^{<\omega}(\mathbb{N}) \backslash \mathcal{F}
$$

For the proof of the block-Ramsey partition theorem we will make use of a diagonal argument, contained in the following lemma.

Lemma 2.5. Let $\mathcal{D}_{0}=\left(s_{n}^{0}\right)_{n \in \mathbb{N}} \in \mathcal{B}^{\omega}(\mathbb{N})$ and $\mathbb{N}\}$.

$\Pi=\left\{(s, \mathcal{D}): s \in[\mathbb{N}]_{>0}^{<\omega}, \mathcal{D}=\left(s_{n}\right)_{n \in \mathbb{N}} \in \mathcal{B}^{\omega}(\mathbb{N})\right.$ with $\mathcal{D}<\mathcal{D}_{0}$ and $s<s_{n} \forall n \in$

If a subset $\mathcal{G}$ of $\Pi$ satisfies:

(i) for every $(s, \mathcal{D}) \in \Pi$ there exists $\left(s, \mathcal{D}_{1}\right) \in \mathcal{G}$ with $\mathcal{D}_{1}<\mathcal{D}$; and

(ii) for every $\left(s, \mathcal{D}_{1}\right) \in \mathcal{G}$ and $\mathcal{D}_{2}<\mathcal{D}_{1}$ we have $\left(s, \mathcal{D}_{2}\right) \in \mathcal{G}$,

then there exists $\mathcal{D} \in \mathcal{B}^{\omega}(\mathbb{N})$ with $\mathcal{D}<\mathcal{D}_{0}$ such that $\left(s, \mathcal{D}^{\prime}\right) \in \mathcal{G}$ for every $s \in F U(\mathcal{D})$ and $\mathcal{D}^{\prime}<\mathcal{D}-s$.

Proof. Let $s_{0}=s_{1}^{0}$. According to condition (i), there exists $\mathcal{D}_{1}=\left(s_{n}^{1}\right)_{n \in \mathbb{N}} \in \mathcal{B}^{\omega}(\mathbb{N})$ with $\mathcal{D}_{1}<\mathcal{D}_{0}-s_{0}$ such that $\left(s_{0}, \mathcal{D}_{1}\right) \in \mathcal{G}$. Let $s_{1}=s_{1}^{1} \in \mathcal{D}_{1}$. Of course, $s_{0}<s_{1}$. We assume that there have been constructed $\mathcal{D}_{1}, \ldots, \mathcal{D}_{n} \in \mathcal{B}^{\omega}(\mathbb{N})$ with $\mathcal{D}_{n}<\cdots<$ $\mathcal{D}_{1}<\mathcal{D}_{0}$ and nonempty finite sets $s_{0}, s_{1}, \ldots, s_{n}$ with $s_{0}<s_{1}<\cdots<s_{n}$ such that for each $1 \leq i \leq n,\left(s, \mathcal{D}_{i}\right) \in \mathcal{G}$ for all $s \in F U\left(\left(s_{0}, \ldots, s_{i-1}\right)\right)$.

We will construct $\mathcal{D}_{n+1}$ and $s_{n+1}$. Let $\left\{t_{1}, \ldots, t_{k}\right\}=F U\left(\left(s_{0}, \ldots, s_{n}\right)\right)$. According to condition (i), there exist $\mathcal{D}_{n+1}^{1}, \ldots, \mathcal{D}_{n+1}^{k} \in \mathcal{B}^{\omega}(\mathbb{N})$ such that $\mathcal{D}_{n+1}^{k}<\cdots<$ $\mathcal{D}_{n+1}^{1}<\mathcal{D}_{n}-s_{n}$ and $\left(t_{i}, \mathcal{D}_{n+1}^{i}\right) \in \mathcal{G}$ for every $1 \leq i \leq k$. Set $\mathcal{D}_{n+1}=\mathcal{D}_{n+1}^{k}$. If $\mathcal{D}_{n+1}=\left(s_{i}^{n+1}\right)_{i \in \mathbb{N}}$ set $s_{n+1}=s_{1}^{n+1}$. Of course $s_{n}<s_{n+1}$. According to condition (ii), $\left(t_{i}, \mathcal{D}_{n+1}\right) \in \mathcal{G}$ for all $1 \leq i \leq k$.

Set $\mathcal{D}=\left(s_{0}, s_{1}, s_{2}, \ldots\right) \in \mathcal{B}^{\omega}(\mathbb{N})$. Then $\mathcal{D}<\mathcal{D}_{0}$. Let $s \in F U(\mathcal{D})$ and $\mathcal{D}^{\prime}<\mathcal{D}-s$. Then $s \in F U\left(\left(s_{0}, s_{1}, \ldots, s_{n_{0}}\right)\right)$, where $n_{0}=\min \left\{n \in \mathbb{N}: s \in F U\left(\left(s_{0}, s_{1}, \ldots, s_{n}\right)\right)\right\}$. Thus $\left(s, \mathcal{D}_{n_{0}+1}\right) \in \mathcal{G}$ and according to (ii), $\left(s, \mathcal{D}^{\prime}\right) \in \mathcal{G}$.

We are now ready to state and prove the block Ramsey partition theorem for every countable ordinal number.

Theorem 2.6 (Block-Ramsey partition theorem for every countable ordinal). Let $\mathcal{F} \subseteq \mathcal{B}^{<\omega}(\mathbb{N})$ be a family of finite-ordered disjoint collections, $\mathcal{D}_{0} \in \mathcal{B}^{\omega}(\mathbb{N})$ an infinite-ordered disjoint collection and $\xi$ a countable ordinal. Then there exists an infinite-ordered disjoint collection $\mathcal{D}<\mathcal{D}_{0}$ such that

$$
\text { either } \mathcal{B}^{\xi} \cap \mathcal{B}^{<\omega}(\mathcal{D}) \subseteq \mathcal{F} \text { or } \mathcal{B}^{\xi} \cap \mathcal{B}^{<\omega}(\mathcal{D}) \subseteq \mathcal{B}^{<\omega}(\mathbb{N}) \backslash \mathcal{F}
$$


Proof. For $\xi=1$ it follows from Theorem 2.4.

Let $\xi>1$. Assume that the theorem is valid for every $\zeta<\xi$. Let $s$ be a nonempty, finite subset of $\mathbb{N}$ with $\min s=n$ and $\mathcal{D} \in \mathcal{B}^{\omega}(\mathbb{N})$ with $\mathcal{D}<\mathcal{D}_{0}$. According to Proposition 2.3, there exist $\xi_{n}<\xi$ such that $\mathcal{B}^{\xi}(s)=\mathcal{B}^{\xi_{n}} \cap\left(\mathcal{B}^{<\omega}(\mathbb{N})-s\right)$. Using the induction hypothesis, there exists $\mathcal{D}_{1} \in \mathcal{B}^{\omega}(\mathbb{N})$ with $\mathcal{D}_{1}<\mathcal{D}$ such that

$$
\text { either } \mathcal{B}^{\xi_{n}} \cap \mathcal{B}^{<\omega}\left(\mathcal{D}_{1}\right) \subseteq \mathcal{F}(s) \quad \text { or } \quad \mathcal{B}^{\xi_{n}} \cap \mathcal{B}^{<\omega}\left(\mathcal{D}_{1}\right) \subseteq \mathcal{B}^{<\omega}(\mathbb{N}) \backslash \mathcal{F}(s) \text {. }
$$

Set $\mathcal{D}_{s}=\mathcal{D}_{1}-s<\mathcal{D}$. Then

$$
\text { either } \mathcal{B}^{\xi}(s) \cap \mathcal{B}^{<\omega}\left(\mathcal{D}_{s}\right) \subseteq \mathcal{F}(s) \quad \text { or } \quad \mathcal{B}^{\xi}(s) \cap \mathcal{B}^{<\omega}\left(\mathcal{D}_{s}\right) \subseteq \mathcal{B}^{<\omega}(\mathbb{N}) \backslash \mathcal{F}(s) .
$$

Let $\mathcal{G}=\left\{(s, \mathcal{D}): s \in[\mathbb{N}]_{>0}^{<\omega}, \mathcal{D}=\left(s_{n}\right)_{n \in \mathbb{N}} \in \mathcal{B}^{\omega}(\mathbb{N})\right.$ with $\mathcal{D}<\mathcal{D}_{0}, s<s_{n}$ for all $n \in \mathbb{N}$ and either $\mathcal{B}^{\xi}(s) \cap \mathcal{B}^{<\omega}(\mathcal{D}) \subseteq \mathcal{F}(s)$ or $\left.\mathcal{B}^{\xi}(s) \cap \mathcal{B}^{<\omega}(\mathcal{D}) \subseteq \mathcal{B}^{<\omega}(\mathbb{N}) \backslash \mathcal{F}(s)\right\}$.

The family $\mathcal{G}$ satisfies conditions (i) (by the above arguments) and (ii) (obviously) of Lemma 2.5. Hence there exists $\mathcal{D}_{1} \in \mathcal{B}^{\omega}(\mathbb{N})$ with $\mathcal{D}_{1}<\mathcal{D}_{0}$ such that $\left(s, \mathcal{D}_{1}^{\prime}\right) \in \mathcal{G}$ for every $s \in F U\left(\mathcal{D}_{1}\right)$ and $\mathcal{D}_{1}^{\prime}<\mathcal{D}_{1}-s$.

Let $\mathcal{F}_{1}=\left\{(s) \in F U\left(\mathcal{D}_{1}\right): \mathcal{B}^{\xi}(s) \cap \mathcal{B}^{<\omega}\left(\mathcal{D}_{1}-s\right) \subseteq \mathcal{F}(s)\right\}$. We use the induction hypothesis for $\xi=1$ (Theorem 2.4). Then there exists $\mathcal{D} \in \mathcal{B}^{\omega}(\mathbb{N})$ with $\mathcal{D}<\mathcal{D}_{1}$ such that

$$
\text { either } \mathcal{B}^{1} \cap \mathcal{B}^{<\omega}(\mathcal{D}) \subseteq \mathcal{F}_{1} \quad \text { or } \quad \mathcal{B}^{1} \cap \mathcal{B}^{<\omega}(\mathcal{D}) \subseteq \mathcal{B}^{<\omega}(\mathbb{N}) \backslash \mathcal{F}_{1}
$$

Since $\mathcal{D}<\mathcal{D}_{1}$ we have $\mathcal{B}^{<\omega}(\mathcal{D}) \subseteq \mathcal{B}^{<\omega}\left(\mathcal{D}_{1}\right)$ and consequently that $(s, \mathcal{D}-s) \in \mathcal{G}$ for every $s \in F U(\mathcal{D})$. Thus

$$
\begin{aligned}
& \text { either } \mathcal{B}^{\xi}(s) \cap \mathcal{B}^{<\omega}(\mathcal{D}-s) \subseteq \mathcal{F}(s) \text { for every } s \in F U(\mathcal{D}) \\
& \text { or } \mathcal{B}^{\xi}(s) \cap \mathcal{B}^{<\omega}(\mathcal{D}-s) \subseteq \mathcal{B}^{<\omega}(\mathbb{N}) \backslash \mathcal{F}(s) \text { for every } s \in F U(\mathcal{D}) .
\end{aligned}
$$

Hence,

$$
\text { either } \mathcal{B}^{\xi} \cap \mathcal{B}^{<\omega}(\mathcal{D}) \subseteq \mathcal{F} \quad \text { or } \quad \mathcal{B}^{\xi} \cap \mathcal{B}^{<\omega}(\mathcal{D}) \subseteq \mathcal{B}^{<\omega}(\mathbb{N}) \backslash \mathcal{F} .
$$

The case $\xi=\omega$ of Theorem 2.6 is the following:

Corollary 2.7. Let $\mathcal{D}_{0} \in \mathcal{B}^{\omega}(\mathbb{N})$ and $\mathcal{F} \subseteq\left[F U\left(\mathcal{D}_{0}\right)\right]_{>0}^{<\omega}$. Then, there exists an infinite sequence $\mathcal{D}=\left(s_{n}\right)_{n \in \mathbb{N}} \subseteq F U\left(\mathcal{D}_{0}\right)$ with $s_{n}<s_{n+1}$ for all $n \in \mathbb{N}$ such that

$$
\begin{aligned}
& \text { either }\left\{\left\{s_{1}, \ldots, s_{k}\right\} \subseteq F U(\mathcal{D}): k \in \mathbb{N} \text { and } \min \bigcup_{i=1}^{k} s_{i}=k\right\} \subseteq \mathcal{F} \\
& \text { or }\left\{\left\{s_{1}, \ldots, s_{k}\right\} \subseteq F U(\mathcal{D}): k \in \mathbb{N} \text { and } \min \bigcup_{i=1}^{k} s_{i}=k\right\} \subseteq\left[F U\left(\mathcal{D}_{0}\right)\right]_{>0}^{<\omega} \backslash \mathcal{F} .
\end{aligned}
$$

For completeness' sake we now state the corresponding result for sum-sets.

Definition 2.8. (i) The natural correspondence between $\mathbb{N}$ and $[\mathbb{N}]<\omega$ is given by the function $\varphi:[\mathbb{N}]^{<\omega} \rightarrow \mathbb{N}$ with $\varphi(s)=\sum_{\ell \in s} 2^{\ell-1}$ for $s \in[\mathbb{N}]^{<\omega}$.

(ii) For every $n \in \mathbb{N}$ we define $c(n)=\min \varphi^{-1}(n)$.

(iii) For every countable ordinal $1 \leq \xi<\omega_{1}$, we define the $\xi$-sum set $\mathcal{P}^{\xi}(L)$ for every infinite subset $L$ of $\mathbb{N}$ as follows:

$$
\begin{gathered}
\mathcal{P}^{\xi}(L)=\left\{\left(\sum_{\ell \in s_{1}} \ell, \ldots, \sum_{\ell \in s_{k}} \ell\right): k \in \mathbb{N}, s_{1}<\cdots<s_{k} \in[L]_{>0}^{<\omega}\right. \\
\text { and } \left.\left(c\left(\min s_{1}\right), \ldots, c\left(\min s_{k}\right)\right) \in \mathcal{A}_{\xi}\right\} .
\end{gathered}
$$

Corollary 2.9. Let $\mathcal{F} \subseteq[\mathbb{N}]^{<\omega}$ and let $\xi$ be a countable ordinal. Then there exists $L \in[\mathbb{N}]$ such that

$$
\text { either } \mathcal{P}^{\xi}(L) \subseteq \mathcal{F} \quad \text { or } \quad \mathcal{P}^{\xi}(L) \subseteq[\mathbb{N}]^{<\omega} \backslash \mathcal{F} .
$$


Strengthened forms of Theorem 2.6 are given in Section 4 below for partitions which are either hereditary families (in Theorem 4.4) or trees (in Theorem 4.2 and in Theorem 4.6).

\section{Some properties of the ReCURSIVE Block Schreier System}

The main results proved in this section concern the thinness of the families $\mathcal{B}^{\xi}$ (Proposition 3.3), the canonical representation of every ordered disjoint collection with respect to $\mathcal{B}^{\xi}$ (Proposition 3.5), and the computation of the strong CantorBendixson index of $\mathcal{B}^{\xi}$ (Proposition 3.11). These properties, and their proofs, for $\mathcal{B}^{\xi}$, are analogous to those for $\mathcal{A}_{\xi}$ in [F3]; they are necessary for the proof of the main results in Section 4 .

Definition 3.1. Let $\mathcal{F} \subseteq \mathcal{B}^{<\omega}(\mathbb{N})$ be a family of finite disjoint collections:

(i) $\mathcal{F}$ is thin if there are no elements $\mathbf{s}, \mathbf{t} \in \mathcal{F}$ with $\mathbf{s} \neq \mathbf{t}$ and $\mathbf{s} \propto \mathbf{t}$.

(ii) $\mathcal{F}^{*}=\left\{\mathbf{t} \in \mathcal{B}^{<\omega}(\mathbb{N}): \mathbf{t} \propto \mathbf{s}\right.$ for some $\left.\mathbf{s} \in \mathcal{F}\right\} \cup\{\emptyset\}$.

(iii) $\mathcal{F}_{*}=\left\{\mathbf{t} \in \mathcal{B}^{<\omega}(\mathbb{N}): \mathbf{t} \subseteq F U(\mathbf{s})\right.$ for some $\left.\mathbf{s} \in \mathcal{F}\right\} \cup\{\emptyset\}$.

(iv) $\mathcal{F}$ is a tree if $\mathcal{F}^{*}=\mathcal{F}$.

(v) $\mathcal{F}$ is hereditary if $\mathcal{F}_{*}=\mathcal{F}$.

Thinness and canonical representation are two properties of the Schreier system $\left(\mathcal{A}_{\xi}\right)_{\xi<\omega_{1}}$ that were proved useful in F3. We will prove that the block Schreier system $\left(\mathcal{B}^{\xi}\right)_{\xi<\omega_{1}}$ satisfies these properties, too.

Proposition 3.2. (i) (Thinness of $\mathcal{A}_{\xi}$, F3, Proposition 2.2]). The families $\mathcal{A}_{\xi}$, $\xi<\omega_{1}$, are thin (i.e., there are no elements $s, t \in \mathcal{A}_{\xi}$ with $s \propto t$ and $s \neq t$ ).

(ii) (Canonical representation w.r.t. $\mathcal{A}_{\xi}$, [F3, Proposition 2.4]). Every (finite or infinite) nonempty subset of $\mathbb{N}$ has canonical representation with respect to $\mathcal{A}_{\xi}$ (i.e., if $I \in[\mathbb{N}]$, then there exists a unique sequence $\left(s_{n}\right)_{n \in \mathbb{N}} \subseteq \mathcal{A}_{\xi}$ such that $I=\bigcup_{n=1}^{\infty} s_{n}$ and $s_{1}<s_{2}<\cdots$, and if $s \in[\mathbb{N}]_{>0}^{<\omega}$, then either $s$ is a proper initial segment of some element of $\mathcal{A}_{\xi}$ or there exist unique $n \in \mathbb{N}, s_{1}, \ldots, s_{n} \in \mathcal{A}_{\xi}$ and a, possible empty, set $s_{n+1}$ which is a proper initial segment of some element of $\mathcal{A}_{\xi}$ such that $\left.s=\bigcup_{i=1}^{n+1} s_{i}\right)$.

Proposition 3.3. Every family $\mathcal{B}^{\xi}$, for $\xi<\omega_{1}$ is thin.

Proof. The family $\mathcal{B}^{1}=\left\{(s): s \in[\mathbb{N}]^{<\omega}\right\}$ is obviously thin. Let $\xi>1$. Then $\emptyset \notin \mathcal{B}^{\xi}$. Let $\mathbf{s}=\left(s_{1}, \ldots, s_{k}\right) \in \mathcal{B}^{\xi}, \mathbf{t}=\left(t_{1}, \ldots, t_{\lambda}\right) \in \mathcal{B}^{\xi}$ such that $\mathbf{s} \propto \mathbf{t}$. Then $s=\left\{\min s_{1}, \ldots, \min s_{k}\right\} \in \mathcal{A}_{\xi}, t=\left\{\min t_{1}, \ldots, \min t_{\lambda}\right\} \in \mathcal{A}_{\xi}$ and $s \propto t$. Since $\mathcal{A}_{\xi}$ is thin (Proposition $3.2($ ii)), we have that $s=t$ and consequently that $\mathbf{s}=\mathbf{t}$. Hence, the family $\mathcal{B}^{\xi}$ is thin.

In the following we will prove that every ordered disjoint collection (finite or infinite) has unique canonical representation with respect to each family $\mathcal{B}^{\xi}$.

Definition 3.4. Let $\xi$ be a nonzero countable ordinal number.

(i) An element $\mathcal{D}=\left(s_{n}\right)_{n \in \mathbb{N}}$ of $\mathcal{B}^{\omega}(\mathbb{N})$ has canonical representation with respect to $\mathcal{B}^{\xi}$ if there exists a unique strictly increasing sequence $\left(m_{n}\right)_{n \in \mathbb{N}}$ in $\mathbb{N}$ so that $\left(s_{1}, \ldots, s_{m_{1}}\right) \in \mathcal{B}^{\xi}$ and $\left(s_{m_{n-1}+1}, \ldots, s_{m_{n}}\right) \in \mathcal{B}^{\xi}$ for every $n>1$.

(ii) A nonempty element $\mathbf{s}=\left(s_{1}, \ldots, s_{k}\right)$ of $\mathcal{B}^{<\omega}(\mathbb{N})$ has canonical representation with respect to $\mathcal{B}^{\xi}$ if either $\mathbf{s} \in\left(\mathcal{B}^{\xi}\right)^{*} \backslash \mathcal{B}^{\xi}$ or there exist unique $n \in \mathbb{N}$, and $m_{1}, \ldots, m_{n} \in \mathbb{N}$ with $m_{1}<\ldots<m_{n} \leq k$ so that $\left(s_{1}, \ldots, s_{m_{1}}\right), \ldots,\left(s_{m_{n-1}+1}, \ldots\right.$, $\left.s_{m_{n}}\right) \in \mathcal{B}^{\xi}$ and $\left(s_{m_{n}+1}, \ldots, s_{k}\right) \in\left(\mathcal{B}^{\xi}\right)^{*} \backslash \mathcal{B}^{\xi}$, in case $m_{n}<k$. 
Proposition 3.5. Let $\xi$ be a nonzero countable ordinal number. Every nonempty ordered disjoint collection (finite or infinite) has canonical representation with respect to $\mathcal{B}^{\xi}$.

Proof. (i) Let $\mathcal{D}=\left(s_{n}\right)_{n \in \mathbb{N}} \in \mathcal{B}^{\omega}(\mathbb{N})$ be an infinite-ordered disjoint collection. For each $n \in \mathbb{N}$ let $k_{n}=\min s_{n} \in \mathbb{N}$. Of course, $k_{n}<k_{n+1}$ for every $n \in \mathbb{N}$. The set $I=\left\{k_{n}: n \in \mathbb{N}\right\}$ has canonical representation with respect to $\mathcal{A}_{\xi}$ (Proposition [3.2(iii)). Hence, there exists a unique sequence $\left(F_{n}\right)_{n \in \mathbb{N}}$ in $\mathcal{A}_{\xi}$ such that $I=\bigcup_{n=1}^{\infty} F_{n}$ and $F_{1}<F_{2}<\cdots$. Let $\max F_{n}=k_{m_{n}}$ for every $n \in \mathbb{N}$. The sequence $\left(m_{n}\right)_{n \in \mathbb{N}}$ has the property that $\left(s_{1}, \ldots, s_{m_{1}}\right) \in \mathcal{B}^{\xi}$ and $\left(s_{m_{n-1}+1}, \ldots, s_{m_{n}}\right) \in \mathcal{B}^{\xi}$ for every $n>1$. It is the unique sequence with this property, since $\mathcal{B}^{\xi}$ is a thin family.

(ii) Let $\mathbf{s}=\left(s_{1}, \ldots, s_{k}\right) \in \mathcal{B}^{<\omega}(\mathbb{N})$ be a nonempty finite-ordered disjoint collection. Set $s_{k+i}=\left\{n_{k}+i\right\}$ for every $i \in \mathbb{N}$, where $n_{k}=\max s_{k}$. The infinite-ordered disjoint collection $\mathcal{D}=\left(s_{n}\right)_{n \in \mathbb{N}}$ has canonical representation with respect to $\mathcal{B}^{\xi}$, according to (i). Using this fact, it is easy to prove that $\mathbf{s}$ has canonical representation with respect to $\mathcal{B}^{\xi}$. It is unique, since $\mathcal{B}^{\xi}$ is thin.

Identifying every collection $\mathrm{D}$ of finite subsets of $\mathbb{N}$ with its characteristic function $x_{D} \in\{0,1\}^{[\mathbb{N}]^{<\omega}}$, we topologize the set of all the collections of finite subsets of $\mathbb{N}$ by the topology of pointwise convergence (equivalently by the product topology of $\left.\{0,1\}^{[\mathbb{N}]^{<\omega}}\right)$. For every $\mathbf{s}=\left(s_{1}, \ldots, s_{k}\right) \in \mathcal{B}^{<\omega}(\mathbb{N})$ let $\sigma(\mathbf{s})=\left\{s_{1}, \ldots, s_{k}\right\}$, and respectively for every $\mathcal{D}=\left(s_{n}\right)_{n \in \mathbb{N}} \in \mathcal{B}^{\omega}(\mathbb{N})$ let $\sigma(\mathcal{D})=\left\{s_{n}: n \in \mathbb{N}\right\}$ and $\sigma(\emptyset)=\emptyset$.

We will say that a family $\mathcal{F} \subseteq \mathcal{B}^{<\omega}(\mathbb{N})$ is pointwise closed iff the family $\left\{x_{\sigma(\mathbf{s})}: \mathbf{s} \in \mathcal{F}\right\}$ is closed in the topology of pointwise convergence and also that a family $\mathcal{U} \subseteq \mathcal{B}^{\omega}(\mathbb{N})$ is pointwise closed iff $\left\{x_{\sigma(\mathcal{D})}: \mathcal{D} \in \mathcal{U}\right\}$ is pointwise closed in $\{0,1\}^{[\mathbb{N}]^{<\omega}}$.

We next turn our attention to trees and hereditary subfamilies of $B^{<\omega}(\mathbb{N})$.

Proposition 3.6. Let $\mathcal{F} \subseteq \mathcal{B}^{<\omega}(\mathbb{N})$.

(i) If $\mathcal{F}$ is a tree, then $\mathcal{F}$ is pointwise closed if and only if there does not exist an infinite sequence $\left(\mathbf{s}_{n}\right)_{n \in \mathbb{N}}$ in $\mathcal{F}$ such that $\mathbf{s}_{n} \propto \mathbf{s}_{n+1}$ and $\mathbf{s}_{n} \neq \mathbf{s}_{n+1}$ for all $n \in \mathbb{N}$.

(ii) If $\mathcal{F}$ is hereditary, then $\mathcal{F}$ is pointwise closed if and only if there does not exist $\mathcal{D} \in \mathcal{B}^{\omega}(\mathbb{N})$ such that $\mathcal{B}^{<\omega}(\mathcal{D}) \subseteq \mathcal{F}$.

Proof. (i) Let $\mathcal{F}$ be a tree. If there exists $\left(\mathbf{s}_{n}\right)_{n \in \mathbb{N}} \subseteq \mathcal{F}$ such that $\mathbf{s}_{n} \propto \mathbf{s}_{n+1}$ and $\mathbf{s}_{n} \neq \mathbf{s}_{n+1}$ for all $n \in \mathbb{N}$, then the sequence $\left(x_{\sigma\left(\mathbf{s}_{n}\right)}\right)_{n \in \mathbb{N}}$ converges, in the topology of pointwise convergence, to $x_{D}$, where $D=\bigcup_{n \in \mathbb{N}} \mathbf{s}_{n}$. Since $\mathcal{D}$ is an infinite set, the family $\mathcal{F}$ is not pointwise closed.

We assume now that there does not exist a sequence $\left(\mathbf{s}_{n}\right)_{n \in \mathbb{N}} \subseteq \mathcal{F}$ with $\mathbf{s}_{n} \propto \mathbf{s}_{n+1}$ and $\mathbf{s}_{n} \neq \mathbf{s}_{n+1}$ for all $n \in \mathbb{N}$. We will prove that $\mathcal{F}$ is pointwise closed. Let $\left(\mathbf{t}_{i}\right)_{i \in \mathbb{N}} \subseteq \mathcal{F}$ such that the sequence $\left(x_{\sigma\left(\mathbf{t}_{i}\right)}\right)_{i \in \mathbb{N}}$ converges pointwise to $x_{A}$ for some $A \subseteq[\mathbb{N}]^{<\omega}$. Let $t_{1}, t_{2} \in A$. Then, there exists $\iota_{0} \in \mathbb{N}$ such that $\left\{t_{1}, t_{2}\right\} \subseteq \sigma\left(\mathbf{t}_{\iota_{0}}\right)$, hence either $t_{1}<t_{2}$ or $t_{2}<t_{1}$. Thus $A$ is an ordered disjoint collection. If $A=\emptyset$, then $A \in \mathcal{F}$, since $\mathcal{F}$ is a tree. If $A=\left\{t_{1}, \ldots, t_{k}\right\}$ for some $k \in \mathbb{N}$, then $A=\sigma(\mathbf{s})$ for $\mathbf{s}=\left(t_{1}, \ldots, t_{k}\right) \in \mathcal{B}^{<\omega}(\mathbb{N})$. Since $F U\left(\left(\{1\}, \ldots,\left\{\max t_{k}\right\}\right)\right)$ is a finite set, there exists $\iota_{0} \in \mathbb{N}$ such that $\mathbf{s} \propto \mathbf{t}_{\iota_{0}}$ and, since $\mathcal{F}$ is a tree, $\mathbf{s} \in \mathcal{F}$.

We will prove that $A$ cannot be an infinite set. Let $A=\left\{t_{n}: n \in \mathbb{N}\right\}$. Then $A=\sigma(\mathcal{D})$, where $\mathcal{D}=\left(t_{n}\right)_{n \in \mathbb{N}} \in \mathcal{B}^{\omega}(\mathbb{N})$. Set $\mathbf{s}_{n}=\left(t_{1}, \ldots, t_{n}\right)$ for every $n \in \mathbb{N}$. Then $\mathbf{s}_{n} \propto \mathbf{s}_{n+1}$ and $\mathbf{s}_{n} \neq \mathbf{s}_{n+1}$ for all $n \in \mathbb{N}$. We will prove that $\left(\mathbf{s}_{n}\right) \subseteq \mathcal{F}$, which is a contradiction to our assumption. Indeed, let $\mathbf{t}_{i}=\left(t_{1}^{i}, \ldots, t_{n_{i}}^{i}\right)$ for every $i \in \mathbb{N}$. 
For $n \in \mathbb{N}$ and $i \in \mathbb{N}$ set $\mathbf{t}_{i}^{n}=\emptyset$ if $t_{n}<t_{1}^{i}$ and otherwise $\mathbf{t}_{i}^{n}=\left(t_{1}^{i}, \ldots, t_{k_{i}^{n}}^{i}\right)$, where $k_{i}^{n}=\max \left\{1 \leq n \leq n_{i}: t_{n}^{i} \leq t_{n}\right\}$.

Since $\mathbf{t}_{i}^{n} \in \mathcal{F}^{*}$ and $\mathcal{F}$ is a tree we have that $\mathbf{t}_{i}^{n} \in \mathcal{F}$ for all $n, i \in \mathbb{N}$. The sequence $\left(x_{\sigma\left(\mathbf{t}_{i}^{n}\right)}\right)_{i \in \mathbb{N}}$ converges pointwise to $\left(x_{\sigma\left(\mathbf{s}_{n}\right)}\right)$, where $\mathbf{s}_{n}=\left(t_{1}, \ldots, t_{n}\right)$, since $\left(x_{\sigma\left(\mathbf{t}_{i}\right)}\right)_{i \in \mathbb{N}}$ converges pointwise to $x_{A}$. Hence, by the above arguments $\mathbf{s}_{n} \in \mathcal{F}$ for every $n \in \mathbb{N}$. Thus, $\mathcal{F}$ is pointwise closed.

(ii) Let $\mathcal{F}$ be hereditary. If there exists $\mathcal{D}=\left(s_{n}\right)_{n \in \mathbb{N}} \in \mathcal{B}^{\omega}(\mathbb{N})$ such that $\mathcal{B}^{<\omega}(\mathcal{D}) \subseteq \mathcal{F}$, then $\mathbf{s}_{n}=\left(s_{1}, \ldots, s_{n}\right) \in \mathcal{F}$ for every $n \in \mathbb{N}$. According to case (i), $\mathcal{F}$ is not pointwise closed, since it is a tree. On the other hand, if $\mathcal{F}$ is not pointwise closed, according to (i), there exist $\mathcal{D}=\left(s_{n}\right)_{n \in \mathbb{N}}$ such that $\left(s_{1}, \ldots, s_{n}\right) \in \mathcal{F}$ for every $n \in \mathbb{N}$. Then $\mathcal{B}^{<\omega}(\mathcal{D}) \subseteq \mathcal{F}$, since $\mathcal{F}$ is hereditary.

Corollary 3.7. Let $\mathcal{F} \subseteq \mathcal{B}^{<\omega}(\mathbb{N})$ which is hereditary and pointwise closed. Then, every hereditary family $\mathcal{F}_{1}$ with $\mathcal{F}_{1} \subseteq \mathcal{F}$ is also pointwise closed.

Corollary 3.8. Let $\xi$ be a countable ordinal and let $\mathcal{D} \in \mathcal{B}^{\omega}(\mathbb{N})$. Then the hereditary family $\left(\mathcal{B}^{\xi} \cap \mathcal{B}^{<\omega}(\mathcal{D})\right)_{*}$ is pointwise closed.

Proof. The family $\left(\mathcal{B}^{1} \cap \mathcal{B}^{<\omega}(\mathcal{D})\right)_{*}$ is obviously pointwise closed. Let $\xi>1$, and assume that $\left(\mathcal{B}^{\zeta} \cap \mathcal{B}^{<\omega}(\mathcal{D})\right)_{*}$ is pointwise closed for every $\zeta<\xi$. Assume that $\left(\mathcal{B}^{\xi} \cap \mathcal{B}^{<\omega}(\mathcal{D})\right)_{*}$ is not pointwise closed. According to Proposition 3.6. there exists an infinite disjoint collection $\mathcal{D}_{0}=\left(s_{n}\right)_{n \in \mathbb{N}}$ such that $\mathcal{B}^{<\omega}\left(\mathcal{D}_{0}\right) \subseteq\left(\mathcal{B}^{\xi} \cap \mathcal{B}^{<\omega}(\mathcal{D})\right)_{*}$. Let $n \in \mathbb{N}$. Then, $\mathbf{s}_{n}=\left(s_{1}, \ldots, s_{n}\right) \in\left(\mathcal{B}^{\xi} \cap \mathcal{B}^{<\omega}(\mathcal{D})\right)_{*}$, so there exists $\mathbf{t}=\left(t_{1}, \ldots, t_{\lambda}\right) \in$ $\mathcal{B}^{\xi} \cap \mathcal{B}^{<\omega}(\mathcal{D})$ such that $\mathbf{s}_{n} \subseteq F U(\mathbf{t})$. If $k=\min t_{1}$, then, according to Proposition 2.3. $\left(t_{2}, \ldots, t_{\lambda}\right) \in \mathcal{B}^{\xi_{k}} \cap \mathcal{B}^{<\omega}\left(\mathcal{D}-t_{1}\right)$. Hence, $\left(s_{2}, \ldots, s_{n}\right) \in\left(\mathcal{B}^{\xi_{k}} \cap \mathcal{B}^{<\omega}(\mathcal{D})\right)_{*}$. Since $k \leq \min s_{1}$, there exists $k_{1} \leq \min s_{1}$ such that $\left(s_{2}, \ldots, s_{n}\right) \in\left(\mathcal{B}^{\xi_{k_{1}}} \cap \mathcal{B}^{<\omega}(\mathcal{D})\right)_{*}$ for infinitely many $n \in \mathbb{N}$. This gives that $\left(s_{2}, \ldots, s_{n}\right) \in\left(\mathcal{B}^{\xi_{k_{1}}} \cap \mathcal{B}^{<\omega}(\mathcal{D})\right)_{*}$ for every $n \in \mathbb{N}$. According to Proposition 3.6. the family $\left(\mathcal{B}^{\xi_{k_{1}}} \cap \mathcal{B}^{<\omega}(\mathcal{D})\right)_{*}$ is not pointwise closed. This is a contradiction to the induction hypothesis, since $\xi_{k_{1}}<\xi$.

For hereditary and pointwise closed families $\mathcal{F}$ in $\mathcal{B}^{<\omega}(\mathbb{N})$ the strong CantorBendixson index $s_{\mathcal{D}}(\mathcal{F})$ of $\mathcal{F}$ with respect to an infinite-ordered disjoint collection $\mathcal{D} \in \mathcal{B}^{\omega}(\mathbb{N})$ can be defined. The strong Cantor-Bendixson index corresponding to $\mathcal{B}^{\xi}$ will turn out to be $\xi+1$, on any infinite-ordered disjoint collection.

Definition 3.9. Let $\mathcal{F} \subseteq \mathcal{B}^{<\omega}(\mathbb{N})$ be a hereditary and pointwise closed family. For an infinite-ordered disjoint collection $\mathcal{D}$ we define the strong block Cantor-Bendixson derivatives $(\mathcal{F})_{\mathcal{D}}^{\xi}$ of $\mathcal{F}$ on $\mathcal{D}$ for every $\xi<\omega_{1}$ as follows:

$(\mathcal{F})_{\mathcal{D}}^{\xi}=\left\{\mathbf{s} \in \mathcal{F} \cap \mathcal{B}^{<\omega}(\mathcal{D}):\right.$ the set $\left\{t \in F U(\mathcal{D}):\left(s_{1}, \ldots, s_{k}, t\right) \notin \mathcal{F}\right\}$ for $\mathbf{s}=\left(s_{1}, \ldots, s_{k}\right)$ or the set $\{t \in F U(\mathcal{D}):(t) \notin \mathcal{F}\}$ for $\mathbf{s}=\emptyset$ does not contain an infinite-ordered disjoint collection $\}$.

It is easy to verify that $(\mathcal{F})_{\mathcal{D}}^{1}$ is hereditary, hence pointwise closed (Corollary 3.7). So, we can define for every $\xi>1$ the $\xi$-derivatives of $\mathcal{F}$ recursively as follows:

$$
\begin{aligned}
& (\mathcal{F})_{\mathcal{D}}^{\zeta+1}=\left((\mathcal{F})_{\mathcal{D}}^{\zeta}\right)_{\mathcal{D}}^{1} \text { for all } \zeta<\omega_{1} ; \text { and } \\
& (\mathcal{F})_{\mathcal{D}}^{\xi}=\bigcap_{\beta<\xi}(\mathcal{F})_{\mathcal{D}}^{\beta} \text { for } \xi \text { a limit ordinal } .
\end{aligned}
$$

The strong block Cantor-Bendixson index $s_{\mathcal{D}}(\mathcal{F})$ of $\mathcal{F}$ on $\mathcal{D}$ is the smallest countable ordinal $\xi$ such that $(\mathcal{F})_{\mathcal{D}}^{\xi}=\emptyset$. 
Remark 3.10. (i) The strong block Cantor-Bendixson index $s_{\mathcal{D}}(\mathcal{F})$ of a hereditary and pointwise closed family $\mathcal{F} \subseteq \mathcal{B}^{<\omega}(\mathbb{N})$ on an infinite-ordered disjoint collection $\mathcal{D}$ is a countable successor ordinal less than or equal to the "usual" Cantor-Bendixson index $O(\mathcal{F})$ of $\mathcal{F}$ into $\{0,1\}^{[\mathbb{N}]<\omega}$ (see $[\mathrm{K}]$ ).

(ii) $s_{\mathcal{D}}\left(\mathcal{F} \cap \mathcal{B}^{<\omega}(\mathcal{D})\right)=s_{\mathcal{D}}(\mathcal{F})$.

(iii) $s_{\mathcal{D}}\left(\mathcal{F}_{1}\right) \leq s_{\mathcal{D}}\left(\mathcal{F}_{2}\right)$ if $\mathcal{F}_{1}, \mathcal{F}_{2} \subseteq \mathcal{B}^{<\omega}(\mathbb{N})$ are hereditary and pointwise closed families with $\mathcal{F}_{1} \subseteq \mathcal{F}_{2}$.

(iv) If $\mathbf{s} \in(\mathcal{F})_{\mathcal{D}}^{\xi}$, then $\mathbf{s}_{1} \in(\mathcal{F})_{\mathcal{D}_{1}}^{\xi}$ for every $\mathcal{D}_{1}<\mathcal{D}$, where $\sigma(s) \cap F U\left(\mathcal{D}_{1}\right)=$ $\sigma\left(s_{1}\right)$, since $F U\left(\mathcal{D}_{1}\right) \subseteq F U(\mathcal{D})$.

(v) If $\mathcal{D}_{1}<\mathcal{D}$, then $s_{\mathcal{D}_{1}}(\mathcal{F}) \geq s_{\mathcal{D}}(\mathcal{F})$, according to (iv).

(vi) If $\sigma\left(\mathcal{D}_{1}\right) \backslash \sigma(\mathcal{D})$ is a finite set, then $s_{\mathcal{D}_{1}}(\mathcal{F}) \geq s_{\mathcal{D}}(\mathcal{F})$.

Proposition 3.11. Let $\mathcal{D}$ be an infinite-ordered disjoint collection and let $\mathcal{D}_{1}<\mathcal{D}$. Then

$$
s_{\mathcal{D}_{1}}\left(\left(\mathcal{B}^{\xi} \cap \mathcal{B}^{<\omega}(\mathcal{D})\right)_{*}\right)=\xi+1 \text { for every } \xi<\omega_{1} .
$$

Proof. For every $\xi<\omega_{1}$ the families $\left(\mathcal{B}^{\xi} \cap \mathcal{B}^{<\omega}(\mathcal{D})\right)_{*}$ are pointwise closed (Corollary [3.8). Also, for every $s \in F U(\mathcal{D})$ with $\min s=n$ we have, according to Proposition 2.3. that

$$
\left(\mathcal{B}^{\xi} \cap \mathcal{B}^{<\omega}(\mathcal{D})\right)(s)=\mathcal{B}^{\xi_{n}} \cap \mathcal{B}^{<\omega}(\mathcal{D}-s) \text { for some } \xi_{n}<\xi .
$$

We will prove by induction that $\left(\left(\mathcal{B}^{\xi} \cap \mathcal{B}^{<\omega}(\mathcal{D})\right)_{*}\right)_{\mathcal{D}_{1}}^{\xi}=\{\emptyset\}$ for every $\xi<\omega_{1}$. Of course, $\left(\mathcal{B}^{1} \cap \mathcal{B}^{<\omega}(\mathcal{D})\right)_{*}=\{(s): s \in F U(\mathcal{D})\} \cup\{\emptyset\}$. So $\left(\left(\mathcal{B}^{1} \cap \mathcal{B}^{<\omega}(\mathcal{D})\right)_{*}\right)_{\mathcal{D}_{1}}^{1}=\{\emptyset\}$. Let $\xi>1$ and assume that

$$
\left(\left(\mathcal{B}^{\zeta} \cap \mathcal{B}^{<\omega}(\mathcal{D})\right)_{*}\right)_{\mathcal{D}_{1}}^{\zeta}=\{\emptyset\} \text { for every } \zeta<\xi \text { and } \mathcal{D}_{1}<\mathcal{D} \text {. }
$$

Hence, for every $s \in F U\left(\mathcal{D}_{1}\right)$ with $\min s=n$ and $\mathcal{D}_{1}<\mathcal{D}$ we have that

$$
\left(\left(\mathcal{B}^{\xi} \cap \mathcal{B}^{<\omega}(\mathcal{D})\right)(s)_{*}\right)_{\mathcal{D}_{1}}^{\xi_{n}}=\left(\left(\mathcal{B}^{\xi_{n}} \cap \mathcal{B}^{<\omega}(\mathcal{D}-s)\right)_{*}\right)_{\mathcal{D}_{1}}^{\xi_{n}}=\{\emptyset\} .
$$

This gives that $\left.(s) \in\left(\left(\mathcal{B}^{\xi} \cap \mathcal{B}^{<\omega}(\mathcal{D})\right)_{*}\right)\right)_{\mathcal{D}_{1}}^{\xi_{n}}$. So, if $\xi=\zeta+1$ is a successor ordinal, we have that $(s) \in\left(\left(\mathcal{B}^{\xi} \cap \mathcal{B}^{<\omega}(\mathcal{D})\right)_{*}\right)_{\mathcal{D}_{1}}^{\zeta}$ for every $s \in F U\left(\mathcal{D}_{1}\right)$ and consequently that $\emptyset \in\left(\left(\mathcal{B}^{\xi} \cap \mathcal{B}^{<\omega}(\mathcal{D})\right)_{*}\right)_{\mathcal{D}_{1}}^{\xi}$. If $\xi$ is a limit ordinal, we have that $\emptyset \in\left(\left(\mathcal{B}^{\xi} \cap \mathcal{B}^{<\omega}(\mathcal{D})\right)_{*}\right)_{\mathcal{D}_{1}}^{\xi}$, since $\emptyset \in\left(\left(\mathcal{B}^{\xi} \cap \mathcal{B}^{<\omega}(\mathcal{D})\right)_{*}\right)_{\mathcal{D}_{1}}^{\xi_{n}}$ for every $n \in \mathbb{N}$ and $\sup \xi_{n}=\xi$.

If $\{\emptyset\} \neq\left(\left(\mathcal{B}^{\xi} \cap \mathcal{B}^{<\omega}(\mathcal{D})\right)_{*}\right)_{\mathcal{D}_{1}}^{\xi}$ for a $\mathcal{D}_{1}<\mathcal{D}$, then there exist $D_{2}<D_{1}$ and $s \in F U\left(\mathcal{D}_{2}\right)$ such that $\left(\left(\mathcal{B}^{\xi} \cap \mathcal{B}^{<\omega}(\mathcal{D})\right)(s)_{*}\right)_{\mathcal{D}_{2}}^{\xi} \neq \emptyset$ (see Lemma 2.8 in [F3]). This is a contradiction to the induction hypothesis. Hence, $\left(\left(\mathcal{B}^{\xi} \cap \mathcal{B}^{<\omega}(\mathcal{D})\right)_{*}\right)_{\mathcal{D}_{1}}^{\xi}=\emptyset$.

\section{Block Nash-Williams partition theorems FOR EVERY COUNTABLE ORDINAL}

Let us recall that, according to the block Ramsey theorem (Theorem 2.6), for every countable ordinal $\xi$ and every partition of $\mathcal{F}$ of $\mathcal{B}^{<\omega}(\mathbb{N})$ there exists an infinite disjoint collection $\mathcal{D}$, all of whose $\mathcal{B}^{\xi}$-finite blocks are either in the partition family $\mathcal{F}$ itself or in the complement $\mathcal{B}^{<\omega}(\mathbb{N}) \backslash \mathcal{F}$. However, Theorem 2.6 provides no information on how to decide whether it is in $\mathcal{F}$ or in $\mathcal{B}^{<\omega}(\mathbb{N}) \backslash \mathcal{F}$. We now have at our disposal all the tools that will allow us to obtain for a partition family $\mathcal{F}$ that is hereditary (in Theorem 4.4) or a tree (in Theorem 4.6), a criterion, in terms of the strong Cantor-Bendixson index of $\mathcal{F}$, according to which, if this index is greater 
than $\xi+1$, all $\mathcal{B}^{\xi}$-finite blocks fall in $\mathcal{F}$, and if less than $\xi+1$, in $\mathcal{B}^{<\omega}(\mathbb{N}) \backslash \mathcal{F}$ (albeit in a weaker, nonsymmetrical manner).

It will be observed that the main dichotomy of Theorem 4.4 is nonsymmetric, reflecting the fact that the strong property of hereditariness is assumed for the family $\mathcal{F}$ itself. This type of nonsymmetric dichotomies is characteristic of NashWilliams partition theorems, and in fact, from Theorem 4.4 we will derive in the sequel (Theorem 4.6. Corollaries 4.8 and 4.9) various forms of block Nash-Williams theorems.

First we will give a stronger form of the block Ramsey theorem (Theorem 2.6) in case the partition family is a tree, using the canonical representation of an ordered disjoint collection with respect to each family $\mathcal{B}^{\xi}$.

Proposition 4.1. Let $\mathcal{F} \subseteq \mathcal{B}^{<\omega}(\mathbb{N})$ be a tree, $\mathcal{D} \in \mathcal{B}^{<\omega}(\mathbb{N})$ and $\xi<\omega_{1}$. Then $\mathcal{B}^{\xi} \cap \mathcal{B}^{<\omega}(\mathcal{D}) \subseteq \mathcal{B}^{<\omega}(\mathbb{N}) \backslash \mathcal{F}$ if and only if $\mathcal{F} \cap \mathcal{B}^{<\omega}(\mathcal{D}) \subseteq\left(\mathcal{B}^{\xi}\right)^{*} \backslash \mathcal{B}^{\xi}$.

Proof. Let $\mathcal{B}^{\xi} \cap \mathcal{B}^{<\omega}(\mathcal{D}) \subseteq \mathcal{B}^{<\omega}(\mathbb{N}) \backslash \mathcal{F}$ and $\mathbf{s}=\left(s_{1}, \ldots, s_{k}\right) \in \mathcal{F} \cap \mathcal{B}^{<\omega}(\mathcal{D})$. Then $\mathbf{s}$ has canonical representation with respect to $\mathcal{B}^{\xi}$ (Proposition 3.5), hence either $\mathbf{s} \in\left(\mathcal{B}^{\xi}\right)^{*} \backslash \mathcal{B}^{\xi}$, as required, or there exists $\mathbf{s}_{1} \in \mathcal{B}_{\xi}$ such that $\mathbf{s}_{1} \propto \mathbf{s}$. The second case is impossible. Indeed, since $\mathcal{F}$ is a tree and $\mathbf{s} \in \mathcal{F} \cap \mathcal{B}^{<\omega}(\mathcal{D})$, we have that $\mathbf{s}_{1} \in \mathcal{F} \cap \mathcal{B}^{<\omega}(\mathcal{D}) \cap \mathcal{B}_{\xi}$; a contradiction to our assumption. Hence, $\mathcal{F} \cap \mathcal{B}^{<\omega}(\mathcal{D}) \subseteq$ $\left(\mathcal{B}^{\xi}\right)^{*} \backslash \mathcal{B}^{\xi}$.

It is obvious that $\mathcal{B}^{\xi} \cap \mathcal{B}^{<\omega}(\mathcal{D}) \subseteq \mathcal{B}^{<\omega}(\mathbb{N}) \backslash \mathcal{F}$ if $\mathcal{F} \cap \mathcal{B}^{<\omega}(\mathcal{D}) \subseteq\left(\mathcal{B}^{\xi}\right)^{*} \backslash \mathcal{B}^{\xi}$.

Theorem 4.2. Let $\mathcal{F} \subseteq \mathcal{B}^{<\omega}(\mathbb{N})$ be a tree, $\mathcal{D} \in \mathcal{B}^{<\omega}(\mathbb{N})$ and $\xi<\omega_{1}$. Then there exists an infinite-ordered disjoint collection $\mathcal{D}<\mathcal{D}_{0}$ such that

$$
\text { either } \mathcal{B}^{\xi} \cap \mathcal{B}^{<\omega}(\mathcal{D}) \subseteq \mathcal{F} \text { or } \mathcal{F} \cap \mathcal{B}^{<\omega}(\mathcal{D}) \subseteq\left(\mathcal{B}^{\xi}\right)^{*} \backslash \mathcal{B}^{\xi} \text {. }
$$

Corollary 4.3. Let $\xi_{1}, \xi_{2}$ be countable ordinals with $\xi_{1}<\xi_{2}$. For every infiniteordered disjoint collection $\mathcal{D}$ there exists an infinite-ordered disjoint collection $\mathcal{D}_{1}<$ $\mathcal{D}$ such that

$$
\left(\mathcal{B}^{\xi_{1}}\right)_{*} \cap \mathcal{B}^{<\omega}\left(\mathcal{D}_{1}\right) \subseteq\left(\mathcal{B}^{\xi_{2}}\right)^{*} \backslash \mathcal{B}^{\xi_{2}} .
$$

Proof. Of course $\left(\mathcal{B}^{\xi_{1}}\right)_{*}$ is a tree. According to Theorem 4.2 , for every infiniteordered disjoint collection $\mathcal{D}$ there exists $\mathcal{D}_{1}<\mathcal{D}$ such that either $\mathcal{B}^{\xi_{2}} \cap \mathcal{B}^{<\omega}\left(\mathcal{D}_{1}\right) \subseteq$ $\left(\mathcal{B}^{\xi_{1}}\right)_{*}$ or $\left(\mathcal{B}^{\xi_{1}}\right)_{*} \cap \mathcal{B}^{<\omega}\left(\mathcal{D}_{1}\right) \subseteq\left(\mathcal{B}^{\xi_{2}}\right)^{*} \backslash \mathcal{B}^{\xi_{2}}$. The first alternative is impossible, since in this case, $\xi_{2}+1=s_{\mathcal{D}_{1}}\left(\left(\mathcal{B}^{\xi_{2}} \cap \mathcal{B}^{<\omega}\left(\mathcal{D}_{1}\right)\right)_{*}\right) \leq s_{\mathcal{D}_{1}}\left(\left(\mathcal{B}^{\xi_{1}}\right)_{*}\right)=\xi_{1}+1$, according to Proposition 3.11, a contradiction.

Now, using Theorem [2.6. Theorem 4.2, Proposition [3.6] and the concept of the strong block Cantor-Bendixson index (Proposition 3.11) we state and prove a strengthened form of Theorem 2.6 for partitions families which are hereditary. This strengthened form of Theorem 2.6 can be considered as a strengthened block Nash-Williams type partition theorem for hereditary families if we keep in mind the block Nash-Williams partition theorem in Gowers's reformulation (Corollary 4.9 below).

Theorem 4.4. Let $\mathcal{F} \subseteq \mathcal{B}^{<\omega}(\mathbb{N})$ be a hereditary family of finite-ordered disjoint collections and let $\mathcal{D}_{0} \in \mathcal{B}^{\omega}(\mathbb{N})$ be an infinite-ordered disjoint collection. We have the following cases:

[Case 1] The family $\mathcal{F} \cap \mathcal{B}^{<\omega}(\mathcal{D})$ is not pointwise closed.

Then, there exists $\mathcal{D}_{1}<\mathcal{D}$ such that $\mathcal{B}^{<\omega}\left(\mathcal{D}_{1}\right) \subseteq \mathcal{F}$.

[Case 2] The family $\mathcal{F} \cap \mathcal{B}^{<\omega}(\mathcal{D})$ is pointwise closed. 
Then, setting

$$
\xi_{\mathcal{D}}^{\mathcal{F}}=\sup \left\{s_{\mathcal{D}_{1}}(\mathcal{F}): \mathcal{D}_{1}<\mathcal{D}\right\}
$$

which is a countable ordinal, the following subcases obtain:

2(i) If $\xi+1<\xi_{\mathcal{D}}^{\mathcal{F}}$, then there exists $\mathcal{D}_{2}<\mathcal{D}$ such that

$$
\mathcal{B}^{\xi} \cap \mathcal{B}^{<\omega}\left(\mathcal{D}_{2}\right) \subseteq \mathcal{F}
$$

2(ii) if $\xi+1>\xi_{\mathcal{D}}^{\mathcal{F}}$, then for every $\mathcal{D}_{1}<\mathcal{D}$ there exists $\mathcal{D}_{2}<\mathcal{D}_{1}$ such that

$$
\mathcal{B}^{\xi} \cap \mathcal{B}^{<\omega}\left(\mathcal{D}_{2}\right) \subseteq \mathcal{B}^{<\omega}(\mathbb{N}) \backslash \mathcal{F}
$$

(equivalently $\left.\mathcal{F} \cap \mathcal{B}^{<\omega}\left(\mathcal{D}_{2}\right) \subseteq\left(\mathcal{B}^{\xi}\right)^{*} \backslash \mathcal{B}^{\xi}\right)$; and

2(iii) if $\xi+1=\xi_{\mathcal{D}}^{\mathcal{F}}$, then there exists $\mathcal{D}_{2}<\mathcal{D}$ such that either $\mathcal{B}^{\xi} \cap \mathcal{B}^{<\omega}\left(\mathcal{D}_{2}\right) \subseteq \mathcal{F}$ or $\mathcal{B}^{\xi} \cap \mathcal{B}^{<\omega}\left(\mathcal{D}_{2}\right) \subseteq \mathcal{B}^{<\omega}(\mathbb{N}) \backslash \mathcal{F}$

Both alternatives may materialize.

Proof. [Case 1] If the hereditary family $\mathcal{F} \cap \mathcal{B}^{<\omega}(\mathcal{D})$ is not pointwise closed, then, according to Proposition 3.6 there exists $\mathcal{D}_{1}<\mathcal{D}$ such that $\mathcal{B}^{<\omega}\left(\mathcal{D}_{1}\right) \subseteq \mathcal{F}$.

[Case 2] If the hereditary family $\mathcal{F} \cap \mathcal{B}^{<\omega}(\mathcal{D})$ is pointwise closed, then $\xi_{\mathcal{D}}^{\mathcal{F}}$ is a countable ordinal, since the "usual" Cantor-Bendixson index $O(\mathcal{F})$ of $\mathcal{F}$ into $\{0,1\}^{[\mathbb{N}]^{<\omega}}$ is a countable ordinal (Remark 3.10(i)) and $s_{\mathcal{D}_{1}}(\mathcal{F}) \leq O(\mathcal{F})$ for every $\mathcal{D}_{1}<\mathcal{D}$.

2(i) Let $\xi+1<\xi_{\mathcal{D}}^{\mathcal{F}}$. Then there exists $\mathcal{D}_{1}<\mathcal{D}$ such that $\xi+1<s_{\mathcal{D}_{1}}(\mathcal{F})$. According to Theorem 4.2, there exists $\mathcal{D}_{2}<\mathcal{D}_{1}$ such that

$$
\text { either } \mathcal{B}^{\xi} \cap \mathcal{B}^{<\omega}\left(\mathcal{D}_{2}\right) \subseteq \mathcal{F} \quad \text { or } \quad \mathcal{F} \cap \mathcal{B}^{<\omega}\left(\mathcal{D}_{2}\right) \subseteq\left(\mathcal{B}^{\xi}\right)^{*} \backslash \mathcal{B}^{\xi} \subseteq\left(\mathcal{B}^{\xi}\right)_{*} \text {. }
$$

The second alternative is impossible. Indeed, if $\mathcal{F} \cap \mathcal{B}^{<\omega}\left(\mathcal{D}_{2}\right) \subseteq\left(\mathcal{B}^{\xi}\right)_{*}$, then, according to Proposition 3.11, $s_{\mathcal{D}_{2}}(\mathcal{F})=s_{\mathcal{D}_{2}}\left(\mathcal{F} \cap \mathcal{B}^{<\omega}\left(\mathcal{D}_{2}\right)\right) \leq s_{\mathcal{D}_{2}}\left(\left(\mathcal{B}^{\xi}\right)_{*}\right)=\xi+1$; a contradiction. Hence, $\mathcal{B}^{\xi} \cap \mathcal{B}^{<\omega}\left(\mathcal{D}_{2}\right) \subseteq \mathcal{F}$.

2(ii) Let $\xi+1>\xi_{\mathcal{D}}^{\mathcal{F}}$ and $\mathcal{D}_{1}<\mathcal{D}$. According to the $\xi$-block Ramsey partition theorem (Theorem 2.6), there exists $\mathcal{D}_{2}<\mathcal{D}_{1}$ such that

$$
\text { either } \mathcal{B}^{\xi} \cap \mathcal{B}^{<\omega}\left(\mathcal{D}_{2}\right) \subseteq \mathcal{F} \quad \text { or } \quad \mathcal{B}^{\xi} \cap \mathcal{B}^{<\omega}\left(\mathcal{D}_{2}\right) \subseteq \mathcal{B}^{<\omega}(\mathbb{N}) \backslash \mathcal{F} \text {. }
$$

The first alternative is impossible, Indeed, if $\left(\mathcal{B}^{\xi} \cap \mathcal{B}^{<\omega}\left(\mathcal{D}_{2}\right)\right) \subseteq \mathcal{F}$, then, according to Proposition 3.11 and Remark 3.10, we obtain that

$$
\xi+1=s_{\mathcal{D}_{2}}\left(\left(\mathcal{B}^{\xi} \cap \mathcal{B}^{<\omega}\left(\mathcal{D}_{2}\right)\right)_{*}\right) \leq s_{\mathcal{D}_{2}}(\mathcal{F}) \leq \xi_{\mathcal{D}}^{\mathcal{F}}
$$

a contradiction. Hence, $\mathcal{B}^{\xi} \cap \mathcal{B}^{<\omega}\left(\mathcal{D}_{2}\right) \subseteq \mathcal{B}^{<\omega}(\mathbb{N}) \backslash \mathcal{F}$, and according to Proposition 4.1, $\mathcal{F} \cap \mathcal{B}^{<\omega}\left(\mathcal{D}_{2}\right) \subseteq\left(\mathcal{B}^{\xi}\right)^{*} \backslash \mathcal{B}^{\xi}$.

2(iii) Let $\xi+1=\xi_{\mathcal{D}}^{\mathcal{F}}$. According to Theorem 2.6, there exists $\mathcal{D}_{2}<\mathcal{D}$ such that either $\mathcal{B}^{\xi} \cap \mathcal{B}^{<\omega}\left(\mathcal{D}_{2}\right) \subseteq \mathcal{F}$ or $\mathcal{B}^{\xi} \cap \mathcal{B}^{<\omega}\left(\mathcal{D}_{2}\right) \subseteq \mathcal{B}^{<\omega}(\mathbb{N}) \backslash \mathcal{F}$.

That both alternatives may materialize can be seen by considering two simple examples:

(1) $\mathcal{F}=\left\{\mathbf{s}=\left(s_{1}<\cdots<s_{2 k+1}\right) \in \mathcal{B}^{<\omega}(\mathbb{N}): k \in \mathbb{N}\right.$ and $\left.\min s_{1}=k\right\}$. It is easy to see that $\mathcal{F}_{*}$ is pointwise closed (according to Proposition 3.6) and that $\mathcal{F}(s)=\mathcal{B}^{2 n} \cap \mathcal{B}^{<\omega}(\mathbb{N}-s)$ for every $s \in[\mathbb{N}]^{<\omega}$ with $\min s=n$. Analogously to Proposition 3.11, it can be proved that $s_{\mathcal{D}}\left(\mathcal{F}_{*}\right)=\omega+1$ for every $\mathcal{D} \in \mathcal{B}^{\omega}(\mathbb{N})$. It is now easy to verify that

$$
\mathcal{B}^{\omega} \cap \mathcal{B}^{<\omega}(\mathcal{D}) \subseteq \mathcal{F}_{*} \text { for every } \mathcal{D} \in \mathcal{B}^{\omega}(\mathbb{N}) .
$$

(2) $\mathcal{F}=\left\{\mathbf{s}=\left(s_{1}<\cdots<s_{k}\right) \in \mathcal{B}^{<\omega}(\mathbb{N}): k \in \mathbb{N}\right.$ and $\left.\min s_{1}=2 k\right\}$. The family $\mathcal{F}_{*}$ is pointwise closed, and $s_{\mathcal{D}_{1}}\left(\mathcal{F}_{*}\right)=\omega+1$ for every $\mathcal{D}_{1}<\mathcal{D}$, where $\mathcal{D}$ is an 
infinite-ordered disjoint collection containing sets of even natural numbers. Thus, $\xi_{\mathcal{D}}^{\mathcal{F}}=\omega+1$. It is now easy to see that $\mathcal{B}^{\omega} \cap \mathcal{B}^{<\omega}\left(\mathcal{D}_{1}\right) \subseteq \mathcal{B}^{<\omega}(\mathbb{N}) \backslash \mathcal{F}_{*}$ for every $\mathcal{D}_{1}<\mathcal{D}$, since $\mathcal{F} \cap \mathcal{B}^{<\omega}\left(\mathcal{D}_{1}\right) \subseteq\left(\mathcal{B}^{\omega}\right)^{*} \backslash \mathcal{B}^{\omega}$.

Using Theorem 4.4 and Corollary 4.3, we will state and prove (in Theorem 4.6 below) a strengthened form of Theorem 2.6 and Theorem 4.2 for partitions families which are trees. This strengthened form of Theorem [2.6, as indicated in Remark 4.11 below, is also a stronger form of the block Nash-Williams partition theorem (Corollary [4.10), proved by Milliken [M] and independently by Taylor [T1].

We will need the following definition.

Definition 4.5. Let $\mathcal{F}$ be a family of finite disjoint collections on $\mathbb{N}$. We set

$$
\mathcal{F}_{h}=\left\{\mathbf{s} \in \mathcal{F}: \text { every } \mathbf{t}=\left(t_{1}, \ldots, t_{k}\right) \text { with } \sigma(\mathbf{t}) \subseteq F U(\mathbf{s}) \text { belongs to } \mathcal{F}\right\} \cup\{\emptyset\} .
$$

Of course, $\mathcal{F}_{h}$ is the largest subfamily of $\mathcal{F} \cup\{\emptyset\}$ which is hereditary.

Theorem 4.6 (Stronger form of block Nash-Williams partition theorem). Let $\mathcal{F} \subseteq$ $\mathcal{B}^{<\omega}(\mathbb{N})$ be a family of finite-ordered disjoint collections which is a tree and let $\mathcal{D}_{0} \in \mathcal{B}^{\omega}(\mathbb{N})$ be an infinite-ordered disjoint collection. We have the following cases:

[Case 1] The family $\mathcal{F}_{h} \cap \mathcal{B}^{<\omega}(\mathcal{D})$ is not pointwise closed.

Then, there exists $\mathcal{D}_{1}<\mathcal{D}$ such that $\mathcal{B}^{<\omega}\left(\mathcal{D}_{1}\right) \subseteq \mathcal{F}$.

[Case 2] The family $\mathcal{F}_{h} \cap \mathcal{B}^{<\omega}(\mathcal{D})$ is pointwise closed.

Then setting

$$
\zeta_{\mathcal{D}}^{\mathcal{F}}=\xi_{\mathcal{D}}^{\mathcal{F}_{h}}=\sup \left\{s_{\mathcal{D}_{1}}\left(\mathcal{F}_{h}\right): \mathcal{D}_{1}<\mathcal{D}\right\}
$$

which is a countable ordinal, the following subcases obtain:

2(i) If $\xi+1<\zeta_{\mathcal{D}}^{\mathcal{F}}$, then there exists $\mathcal{D}_{2}<\mathcal{D}$ such that

$$
\mathcal{B}^{\xi} \cap \mathcal{B}^{<\omega}\left(\mathcal{D}_{2}\right) \subseteq \mathcal{F}
$$

2(ii) if $\xi+1>\xi>\zeta_{\mathcal{D}}^{\mathcal{F}}$, then for every $\mathcal{D}_{1}<\mathcal{D}$ there exists $\mathcal{D}_{2}<\mathcal{D}_{1}$ such that

$$
\mathcal{B}^{\xi} \cap \mathcal{B}^{<\omega}\left(\mathcal{D}_{2}\right) \subseteq \mathcal{B}^{<\omega}(\mathbb{N}) \backslash \mathcal{F}
$$

(equivalently $\left.\mathcal{F} \cap \mathcal{B}^{<\omega}\left(\mathcal{D}_{2}\right) \subseteq\left(\mathcal{B}^{\xi}\right)^{*} \backslash \mathcal{B}^{\xi}\right)$; and

2(iii) if $\xi+1=\zeta_{\mathcal{D}}^{\mathcal{F}}$ or $\xi=\zeta_{\mathcal{D}}^{\mathcal{F}}$, then there exists $\mathcal{D}_{2}<\mathcal{D}$ such that either $\mathcal{B}_{\xi} \cap \mathcal{B}^{<\omega}\left(\mathcal{D}_{2}\right) \subseteq \mathcal{F}$ or $\mathcal{B}_{\xi} \cap \mathcal{B}^{<\omega}\left(\mathcal{D}_{2}\right) \subseteq \mathcal{B}^{<\omega}(\mathbb{N}) \backslash \mathcal{F}$.

Proof. [Case 1] If the hereditary family $\mathcal{F}_{h} \cap \mathcal{B}^{<\omega}(\mathcal{D})$ is not pointwise closed, then there exists $\mathcal{D}_{1}<\mathcal{D}$ such that $\mathcal{B}^{<\omega}\left(\mathcal{D}_{1}\right) \subseteq \mathcal{F}_{h} \subseteq \mathcal{F}$, according to Proposition 3.6.

[Case 2] If $\mathcal{F}_{h} \cap \mathcal{B}^{<\omega}(\mathcal{D})$ is pointwise closed, then $\zeta_{\mathcal{D}}^{\mathcal{F}}$ is a countable ordinal, according to Theorem 4.4 .

2(i) Let $\xi+1<\zeta_{\mathcal{D}}^{\mathcal{F}}$. Then $\xi+1<\xi_{\mathcal{D}}^{\mathcal{F}_{h}}$, and, according to Theorem 4.4 (subcase $2(\mathrm{i}))$, there exists $\mathcal{D}_{2}<\mathcal{D}$ such that

$$
\mathcal{B}^{\xi} \cap \mathcal{B}^{<\omega}\left(\mathcal{D}_{2}\right) \subseteq \mathcal{F}_{h} \subseteq \mathcal{F} .
$$

2(ii) Let $\xi+1>\xi>\zeta_{\mathcal{D}}^{\mathcal{F}}$ and $\mathcal{D}_{1}<\mathcal{D}$. According to Theorem 4.4 (subcase 2(ii)), there exists $\mathcal{D}_{1}^{\prime}<\mathcal{D}_{1}$ such that

$$
\mathcal{B}^{\zeta_{\mathcal{D}}^{\mathcal{F}}} \cap \mathcal{B}^{<\omega}\left(\mathcal{D}_{1}^{\prime}\right) \subseteq \mathcal{B}^{<\omega}(\mathbb{N}) \backslash \mathcal{F}_{h}
$$

According to the $\xi$-block Ramsey partition theorem (Theorem 2.6), there exists $\mathcal{D}_{2}<\mathcal{D}_{1}^{\prime}$ such that

$$
\text { either } \mathcal{B}^{\xi} \cap \mathcal{B}^{<\omega}\left(\mathcal{D}_{2}\right) \subseteq \mathcal{F} \quad \text { or } \quad \mathcal{B}^{\xi} \cap \mathcal{B}^{<\omega}\left(\mathcal{D}_{2}\right) \subseteq \mathcal{B}^{<\omega}(\mathbb{N}) \backslash \mathcal{F}
$$


We claim that the first alternative does not hold. Indeed, if $\mathcal{B}^{\xi} \cap \mathcal{B}^{<\omega}\left(\mathcal{D}_{2}\right) \subseteq \mathcal{F}$, then $\left(\mathcal{B}^{\xi} \cap \mathcal{B}^{<\omega}\left(\mathcal{D}_{2}\right)\right)^{*} \subseteq \mathcal{F}^{*}=\mathcal{F}$. Using the canonical representation of every infinite-ordered disjoint collection with respect to $\mathcal{B}_{\xi}$ (Proposition 3.5), it is easy to check that

$$
\left(\mathcal{B}^{\xi}\right)^{*} \cap \mathcal{B}^{<\omega}\left(\mathcal{D}_{2}\right)=\left(\mathcal{B}^{\xi} \cap \mathcal{B}^{<\omega}\left(\mathcal{D}_{2}\right)\right)^{*}
$$

Hence, $\left(\mathcal{B}^{\xi}\right)^{*} \cap \mathcal{B}^{<\omega}\left(\mathcal{D}_{2}\right) \subseteq \mathcal{F}$.

Since $\xi>\zeta_{\mathcal{D}}^{\mathcal{F}}$, according to Corollary 4.3 , there exists $\mathcal{D}_{3}<\mathcal{D}_{2}$ such that $\left(\mathcal{B}^{\zeta_{\mathcal{D}}^{\mathcal{F}}}\right)_{*} \cap$ $\mathcal{B}^{<\omega}\left(\mathcal{D}_{3}\right) \subseteq\left(\mathcal{B}^{\xi}\right)^{*} \cap \mathcal{B}^{<\omega}\left(\mathcal{D}_{2}\right) \subseteq \mathcal{F}$. Thus $\left(\mathcal{B}^{\zeta_{\mathcal{D}}^{\mathcal{F}}}\right)_{*} \cap \mathcal{B}^{<\omega}\left(\mathcal{D}_{3}\right) \subseteq \mathcal{F}_{h}$. This is a contradiction to (11). Hence, $\mathcal{B}^{\xi} \cap \mathcal{B}^{<\omega}\left(\mathcal{D}_{2}\right) \subseteq \mathcal{B}^{<\omega}(\mathbb{N}) \backslash \mathcal{F}$ and equivalently $\mathcal{F} \cap$ $\mathcal{B}^{<\omega}\left(\mathcal{D}_{2}\right) \subseteq\left(\mathcal{B}_{\xi}\right)^{*} \backslash \mathcal{B}_{\xi}$ (Proposition 4.1).

2(iii) In the cases $\zeta_{\mathcal{D}}^{\mathcal{F}}=\xi+1$ or $\zeta_{\mathcal{D}}^{\mathcal{F}}=\xi$, we use Theorem 2.6.

Condition 2(ii) of Theorems 4.4 and 4.6 has a number of interesting reformulations.

Proposition 4.7. Let $\mathcal{F} \subseteq \mathcal{B}^{<\omega}(\mathbb{N}), \xi<\omega_{1}$ and $\mathcal{D} \in \mathcal{B}^{<\omega}(\mathbb{N})$. The following are equivalent:

(i) $\mathcal{B}^{\xi} \cap \mathcal{B}^{<\omega}(\mathcal{D}) \subseteq \mathcal{F}$.

(ii) For every $\mathcal{D}_{1}<\mathcal{D}$ the unique initial segment of $\mathcal{D}_{1}$ which is an element of $\mathcal{B}^{\xi}$ belongs to $\mathcal{F}$.

(iii) Given any sequence $\left(\mathcal{D}_{n}\right)_{n \in \mathbb{N}}$ in $B^{\omega}(\mathbb{N})$ with $\mathcal{D}_{n}<\mathcal{D}$ for every $n \in \mathbb{N}$, and $s_{n} \in F U\left(\mathcal{D}_{n}\right)$ for every $n \in \mathbb{N}$ with $s_{1}<s_{2}<\cdots$, then there exists $n_{0} \in \mathbb{N}$ such that $\left(s_{1}, \ldots, s_{n_{0}}\right) \in \mathcal{B}^{\xi} \cap \mathcal{F}$.

Proof. (i) $\Rightarrow$ (ii). Let $\mathcal{D}_{1}<\mathcal{D}$. Using the canonical representation of $\mathcal{D}_{1}$ with respect to $\mathcal{B}_{\xi}$ (Proposition 3.5), there exists a unique initial segment $\mathbf{s}$ of $\mathcal{D}_{1}$ which is an element of $\mathcal{B}^{\xi}$. Since $\mathbf{s} \in \mathcal{B}^{\xi} \cap \mathcal{B}^{<\omega}(\mathcal{D})$, we have that $\mathbf{s} \in \mathcal{F}$, according to (i).

(ii) $\Rightarrow$ (i). Let $\mathcal{D}=\left(s_{n}\right)_{n \in \mathbb{N}}$ and $\mathbf{s}=\left(t_{1}, \ldots, t_{k}\right) \in \mathcal{B}^{\xi} \cap \mathcal{B}^{<\omega}(\mathcal{D})$. We set $l=\min \left\{n \in \mathbb{N}: s_{n}>t_{k}\right\}$ and $\mathcal{D}_{1}=\left\{t_{1}, \ldots, t_{k}, s_{l}, s_{l+1}, \ldots\right\}$. Of course $\mathcal{D}_{1}<\mathcal{D}$. Using the canonical representation of $\mathcal{D}_{1}$ with respect to $\mathcal{B}_{\xi}$ (Proposition 3.5), and (ii), we have that $\mathbf{s} \in \mathcal{F}$.

(i) $\Rightarrow$ (iii). Let $\left(\mathcal{D}_{n}\right)_{n \in \mathbb{N}}$ be a sequence in $B^{\omega}(\mathbb{N})$ with $\mathcal{D}_{n}<\mathcal{D}$ for every $n \in \mathbb{N}$. Choose $s_{n} \in F U\left(\mathcal{D}_{n}\right)$ for every $n \in \mathbb{N}$ with $s_{1}<s_{2}<\cdots$. The infinite-ordered disjoint collection $\left(s_{n}\right)_{n \in \mathbb{N}}$ has canonical representation with respect to $\mathcal{B}_{\xi}$, hence there exists a unique $n_{0} \in \mathbb{N}$ such that $\left(s_{1}, \ldots, s_{n_{0}}\right) \in \mathcal{B}^{\xi} \cap \mathcal{B}^{<\omega}(\mathcal{D})$. According to (i), we have that $\left(s_{1}, \ldots, s_{n_{0}}\right) \in \mathcal{F}$.

(iii) $\Rightarrow$ (ii). Let $\mathcal{D}_{1}<\mathcal{D}$ and let $\mathcal{D}_{1}=\left(t_{n}\right)_{n \in \mathbb{N}}$. Using (iii) with $\mathcal{D}_{n}=\mathcal{D}_{1}$ for every $n \in \mathbb{N}$ and $s_{n}=t_{n}$ for every $n \in \mathbb{N}$, we have the existence of an $n_{0} \in \mathbb{N}$ such that $\left(t_{1}, \ldots, t_{n_{0}}\right) \in \mathcal{B}^{\xi} \cap \mathcal{F}$.

The following corollary is a simplified statement of Theorem 4.6.

Corollary 4.8. Let $\mathcal{F} \subseteq \mathcal{B}^{<\omega}(\mathbb{N})$, which is a tree, and let $\mathcal{D} \in \mathcal{B}^{\omega}(\mathbb{N})$. Then

(i) either there exists $\mathcal{D}_{1}<\mathcal{D}$ such that $\mathcal{B}^{<\omega}\left(\mathcal{D}_{1}\right) \subseteq \mathcal{F}$,

(ii) or there exists a countable ordinal $\xi_{0}=\zeta_{\mathcal{D}}^{\mathcal{F}}$ such that for all $\xi>\xi_{0}$ and $\mathcal{D}_{1}<\mathcal{D}$, there exists $\mathcal{D}_{2}<\mathcal{D}$ such that $\mathcal{B}^{\xi} \cap \mathcal{B}^{<\omega}\left(\mathcal{D}_{2}\right) \subseteq \mathcal{B}^{<\omega}(\mathbb{N}) \backslash \mathcal{F}$.

Proof. We apply Theorem 4.6 (Case 1 and subcase 2(ii)).

We now consider a very simplified statement of Theorem 4.6 (not including countable ordinals), more akin to the Gowers' reformulation (in $[\mathbf{G}$ ) of the classical Nash-Williams theorem [NW]. 
Corollary 4.9 (Block Nash-Williams partition theorem in Gowers's reformulation). Let $\mathcal{F} \subseteq \mathcal{B}^{<\omega}(\mathbb{N})$, which is a tree, and let $\mathcal{D} \in \mathcal{B}^{\omega}(\mathbb{N})$. Then there exists $\mathcal{D}_{1}<\mathcal{D}$ such that

(i) either $\mathcal{B}^{<\omega}\left(\mathcal{D}_{1}\right) \subseteq \mathcal{F}$,

(ii) or for every $\mathcal{D}_{2}<\mathcal{D}_{1}$ there exists an initial segment of $\mathcal{D}_{2}$ which belongs to $\mathcal{B}^{<\omega}(\mathbb{N}) \backslash \mathcal{F}$.

Proof. The proof follows from Theorem 4.6 (Case 1 and subcase 2(ii)) and Proposition 4.7

Corollary 4.9 implies the following block Nash-Williams theorem, proved by Milliken $[\mathrm{M}$ and independently by Taylor [T1. Moreover, Corollary 4.9 is equivalent to the block Nash-Williams theorem, according to Remark 4.11, This is the reason we called Theorem 4.6 a stronger form of the block Nash-Williams partition theorem.

Corollary 4.10 (Block Nash-Williams theorem, [M], T1]). Let $\mathcal{U} \subseteq \mathcal{B}^{\omega}(\mathbb{N})$ be a pointwise closed family and $\mathcal{D} \in \mathcal{B}^{\omega}(\mathbb{N})$. Then there exists $\mathcal{D}_{1}<\mathcal{D}$ such that

$$
\text { either } \quad \mathcal{B}^{\omega}\left(\mathcal{D}_{1}\right) \subseteq \mathcal{U} \quad \text { or } \quad \mathcal{B}^{\omega}\left(\mathcal{D}_{1}\right) \subseteq \mathcal{B}^{\omega}(\mathbb{N}) \backslash \mathcal{U}
$$

Proof. Let $\mathcal{F}_{\mathcal{U}}=\left\{\mathbf{s} \in \mathcal{B}^{<\omega}(\mathbb{N})\right.$ : there exists $\mathcal{D}^{\prime} \in \mathcal{U}$ such that $\left.\mathbf{s} \propto \mathcal{D}^{\prime}\right\}$. Since the family $\mathcal{F}_{\mathcal{U}}$ is a tree, we use Corollary 4.9 . Then we have the following two cases:

[Case 1] There exists $\mathcal{D}_{1}<\mathcal{D}$ such that $\mathcal{B}^{<\omega}\left(\mathcal{D}_{1}\right) \subseteq \mathcal{F}_{\mathcal{U}}$. Then, $\mathcal{B}^{\omega}\left(\mathcal{D}_{1}\right) \subseteq \mathcal{U}$. Indeed, if $\mathcal{D}_{2}=\left(s_{n}\right)_{n \in \mathbb{N}} \in \mathcal{B}^{\omega}\left(\mathcal{D}_{1}\right)$, then $\left(s_{1}, \ldots, s_{n}\right) \in \mathcal{F}_{\mathcal{U}}$ for every $n \in \mathbb{N}$. Hence, for every $n \in \mathbb{N}$ there exists $\mathcal{D}_{n}^{\prime} \in \mathcal{U}$ such that $\left(s_{1}, \ldots, s_{n}\right) \propto \mathcal{D}_{n}^{\prime}$. Since $\left(x_{\sigma\left(\mathcal{D}_{n}^{\prime}\right)}\right)_{n \in \mathbb{N}}$ converges pointwise to $x_{\sigma\left(\mathcal{D}_{2}\right)}$ and $\mathcal{U}$ is pointwise closed, we have that $\mathcal{D}_{2} \in \mathcal{U}$ and consequently that $\mathcal{B}^{\omega}\left(\mathcal{D}_{1}\right) \subseteq \mathcal{U}$.

[Case 2] There exists $\mathcal{D}_{1}<\mathcal{D}$ such that for every $\mathcal{D}_{2}<\mathcal{D}_{1}$ there exists an initial segment $\left(s_{1}, \ldots, s_{n}\right)$ of $\mathcal{D}_{2}$ which belongs to $\mathcal{B}^{<\omega}(\mathbb{N}) \backslash \mathcal{F}_{\mathcal{U}}$. Then, $\mathcal{D}_{2} \in \mathcal{B}^{\omega}(\mathbb{N}) \backslash \mathcal{U}$ for every $\mathcal{D}_{2}<\mathcal{D}_{1}$. Hence, $\mathcal{B}^{\omega}\left(\mathcal{D}_{1}\right) \subseteq \mathcal{B}^{\omega}(\mathbb{N}) \backslash \mathcal{U}$.

Remark 4.11. (i) The block Nash-Williams partition theorem (Corollary 4.10) is in fact equivalent to Corollary 4.9. Indeed, let $\mathcal{F} \subseteq \mathcal{B}^{<\omega}(\mathbb{N})$. Set

$$
\mathcal{U}_{\mathcal{F}}=\left\{\mathcal{D} \in \mathcal{B}^{<\omega}(\mathbb{N}): \text { there exists }\left(s_{1}, \ldots, s_{k}\right) \in \mathcal{F} \text { such that }\left(s_{1}, \ldots, s_{k}\right) \propto \mathcal{D}\right\} .
$$

The complement $\mathcal{B}^{\omega}(\mathbb{N}) \backslash \mathcal{U}_{\mathcal{F}}$ is pointwise closed, so, using Corollary 4.10 for the family $\mathcal{B}^{\omega}(\mathbb{N}) \backslash \mathcal{U}_{\mathcal{F}}$, we obtain Corollary 4.9. On the other hand, Corollary 4.9 implies Corollary 4.10, according to the proof of Corollary 4.10.

(ii) Corollary 4.9 holds for arbitrary partitions of $\mathcal{B}^{<\omega}(\mathbb{N})$ (not necessarily trees). Indeed, let $\mathcal{F}$ be an arbitrary partition of $\mathcal{B}^{<\omega}(\mathbb{N})$. Then we set $\mathcal{F}_{t}=\{\mathbf{s} \in \mathcal{F}$ : every $\mathbf{t} \propto \mathbf{s}$ belongs to $\mathcal{F}\} \cup\{\emptyset\}$. The family $\mathcal{F}_{t}$ is a tree and $\mathcal{F}_{t} \subseteq \mathcal{F}$. According to Corollary 4.9, there exists $\mathcal{D}_{1}<\mathcal{D}$ such that:

either $\mathcal{B}^{<\omega}\left(\mathcal{D}_{1}\right) \subseteq \mathcal{F}_{t} \subseteq \mathcal{F}$;

or for every $\mathcal{D}_{2}<\mathcal{D}_{1}$ there exists an initial segment of $\mathcal{D}_{2}$ which belongs to $\mathcal{B}^{<\omega}(\mathbb{N}) \backslash \mathcal{F}_{t}$.

Let $\mathcal{D}_{2}<\mathcal{D}_{1}$, and let $\mathbf{s} \propto \mathcal{D}_{2}$ with $\mathbf{s} \in \mathcal{B}^{<\omega}(\mathbb{N}) \backslash \mathcal{F}_{t}=\left(\mathcal{F} \backslash \mathcal{F}_{t}\right) \cup\left(\mathcal{B}^{<\omega}(\mathbb{N}) \backslash \mathcal{F}\right)$. Then, either $\mathbf{s} \in \mathcal{B}^{<\omega}(\mathbb{N}) \backslash \mathcal{F}$, as required, or $\mathbf{s} \in \mathcal{F} \backslash \mathcal{F}_{t}$. In case $\mathbf{s} \in \mathcal{F} \backslash \mathcal{F}_{t}$, by the definition of $\mathcal{F}_{t}$, there exists $\mathbf{s}_{1} \propto \mathbf{s}$ such that $\mathbf{s}_{1} \in \mathcal{B}^{<\omega}(\mathbb{N}) \backslash \mathcal{F}$, as required. 


\section{The Block Ellentuck Partition theorem FOR EVERY COUNTABLE ORDINAL}

In this section we show that our Theorem 4.6 implies a stronger partition theorem, for partitions of $\mathcal{B}^{\omega}(\mathbb{N})$, involving the Ellentuck topology $\mathfrak{T}_{E}$ (Theorem 5.2 ). A simple consequence of Theorem 5.2 (together with Corollary 5.5) is the characterization of completely Ramsey partitions of $\mathcal{B}^{\omega}(\mathbb{N})$ in terms of the Baire property in the topology $\mathfrak{T}_{E}$, a result proved with different methods by Milliken [M] and also by Taylor T1.

We define below the topology $\mathfrak{T}_{E}$ on $\mathcal{B}^{\omega}(\mathbb{N})$, a block analogue of the Ellentuck topology $[\mathrm{E}]$.

Definition 5.1. Let $\mathfrak{T}_{E}$ be the topology on $\mathcal{B}^{\omega}(\mathbb{N})$ with basic open sets of the form

$$
[\mathbf{s}, \mathcal{D}]=\left\{\mathcal{D}_{1} \in \mathcal{B}^{\omega}(\mathbb{N}): \mathbf{s} \propto \mathcal{D}_{1} \quad \text { and } \quad \mathcal{D}_{1}-\mathbf{s}<\mathcal{D}\right\}
$$

where $\mathbf{s} \in \mathcal{B}^{<\omega}(\mathbb{N})$ and $\mathcal{D} \in \mathcal{B}^{\omega}(\mathbb{N})$.

The topology $\mathfrak{T}_{E}$ is stronger than the relative topology of $\mathcal{B}^{<\omega}(\mathbb{N})$ with respect to the pointwise convergence topology of $\{0,1\}^{[\mathbb{N}]^{<\omega}}$, which has basic open sets of the form $[\mathbf{s}, \mathbb{N}]=\left\{\mathcal{D}_{1} \in \mathcal{B}^{\omega}(\mathbb{N}): \mathbf{s} \propto \mathcal{D}_{1}\right\}$.

We denote by $\hat{\mathcal{U}}$ and $\mathcal{U}^{\diamond}$ the closure and the interior, respectively, of a family $\mathcal{U} \subseteq \mathcal{B}^{\omega}(\mathbb{N})$ in the topology $\mathfrak{T}_{E}$. Then it is easy to see that

$$
\begin{aligned}
& \hat{\mathcal{U}}=\left\{\mathcal{D} \in \mathcal{B}^{\omega}(\mathbb{N}):[\mathbf{s}, \mathcal{D}] \cap \mathcal{U} \neq \emptyset \text { for every } \mathbf{s} \propto \mathcal{D}\right\} ; \text { and } \\
& \mathcal{U}^{\diamond}=\left\{\mathcal{D} \in \mathcal{B}^{\omega}(\mathbb{N}): \text { there exists } \mathbf{s} \propto \mathcal{D} \text { such that }[\mathbf{s}, \mathcal{D}] \subseteq \mathcal{U}\right\}
\end{aligned}
$$

If $\mathbf{s}=\left(s_{1}, \ldots, s_{k}\right) \in \mathcal{B}^{<\omega}(\mathbb{N})$ and $\mathbf{t}=\left(t_{1}, \ldots, t_{l}\right) \in \mathcal{B}^{<\omega}(\mathbb{N})$ with $s_{k}<t_{1}$, then we set $\mathbf{s} * \mathbf{t}=\left(s_{1}, \ldots, s_{k}, t_{1}, \ldots, t_{k}\right) \in \mathcal{B}^{<\omega}(\mathbb{N})$.

Theorem 5.2. Let $\mathcal{U} \subseteq \mathcal{B}^{\omega}(\mathbb{N}), \mathbf{s} \in \mathcal{B}^{<\omega}(\mathbb{N})$ and $\mathcal{D} \in \mathcal{B}^{\omega}(\mathbb{N})$. Then

either there exists $\mathcal{D}_{1}<\mathcal{D}$ such that $\left[\mathbf{s}, \mathcal{D}_{1}\right] \subseteq \hat{\mathcal{U}}$, or there exists a countable ordinal $\xi_{0}=\zeta_{(\mathbf{s}, \mathcal{D})}^{\mathcal{U}}$ such that for every $\xi>\xi_{0}$ there exists $\mathcal{D}_{1}<\mathcal{D}-\mathbf{s}$ with $\left[\mathbf{s} * \mathbf{t}, \mathcal{D}_{1}\right] \subseteq \mathcal{B}^{\omega}(\mathbb{N}) \backslash \mathcal{U}$ for all $\mathbf{t} \in \mathcal{B}^{\xi} \cap \mathcal{B}^{<\omega}\left(\mathcal{D}_{1}\right)$.

We will give the proof of this theorem after the following lemma which is analogous to Lemma 2.5 .

Lemma 5.3. Let $\mathcal{G} \subseteq\left\{[\mathbf{s}, \mathcal{D}]: \mathbf{s} \in \mathcal{B}^{<\omega}(\mathbb{N})\right.$ and $\left.\mathcal{D} \in \mathcal{B}^{\omega}(\mathbb{N})\right\}$ with the following two properties:

(i) for every $(\mathbf{s}, \mathcal{D}) \in \mathcal{B}^{<\omega}(\mathbb{N}) \times \mathcal{B}^{\omega}(\mathbb{N})$ there exists $\mathcal{D}_{1}<\mathcal{D}$ such that $\left[\mathbf{s}, \mathcal{D}_{1}\right] \in$ $\mathcal{G}$; and

(ii) for every $\left[\mathbf{s}, \mathcal{D}_{1}\right] \in \mathcal{G}$ and $\mathcal{D}_{2}<\mathcal{D}_{1}$ we have $\left[\mathbf{s}, \mathcal{D}_{2}\right] \in \mathcal{G}$.

Then, for every $(\mathbf{s}, \mathcal{D}) \in \mathcal{B}^{<\omega}(\mathbb{N}) \times \mathcal{B}^{\omega}(\mathbb{N})$ there exists $\mathcal{D}_{0} \in[\mathbf{s}, \mathcal{D}]$ such that $\left[\mathbf{s} * \mathbf{t}, \mathcal{D}_{1}\right] \in \mathcal{G}$ for every $\mathbf{t} \in \mathcal{B}^{<\omega}\left(\mathcal{D}_{0}-\mathbf{s}\right)$ and $\mathcal{D}_{1}<\mathcal{D}_{0}-\mathbf{s}$.

Proof. Let $\mathbf{s}=\left(s_{1}, \ldots, s_{k}\right) \in \mathcal{B}^{<\omega}(\mathbb{N})$ and $\mathcal{D} \in \mathcal{B}^{\omega}(\mathbb{N})$. We can assume that $\mathcal{D}-\mathbf{s}=\mathcal{D}$. According to assumption $(\mathrm{i})$, there exists $\mathcal{D}_{1}<\mathcal{D}$ such that $\left[\mathbf{s}, \mathcal{D}_{1}\right] \in \mathcal{G}$. Assume that $\mathcal{D}_{n}<\cdots<\mathcal{D}_{1} \in \mathcal{B}^{\omega}(\mathbb{N})$ have been constructed and $\mathcal{D}_{n}=\left(s_{i}^{n}\right)_{i \in \mathbb{N}}$ for every $n \in \mathbb{N}$. 
Set $\left\{\mathbf{t}_{1}, \ldots, \mathbf{t}_{r}\right\}=\left\{\mathbf{t} \in \mathcal{B}^{<\omega}(\mathbb{N}): \sigma(\mathbf{t}) \subseteq F U\left(\left\{s_{1}^{1}, \ldots, s_{n}^{n}\right\}\right)\right\}$. According to (i), there exist $\mathcal{D}_{n+1}^{1}<\mathcal{D}_{n}-s_{n}^{n}$ such that $\left[\mathbf{s} * \mathbf{t}_{1}, \mathcal{D}_{n+1}^{1}\right] \in \mathcal{G}, \mathcal{D}_{n+1}^{2}<\mathcal{D}_{n+1}^{1}$ such that $\left[\mathbf{s} * \mathbf{t}_{2}, \mathcal{D}_{n+1}^{2}\right] \in \mathcal{G}$, and finally $\mathcal{D}_{n+1}^{r}<\mathcal{D}_{n}-s_{n}^{n}$ such that $\left[\mathbf{s} * \mathbf{t}_{r}, \mathcal{D}_{n+1}^{r}\right] \in \mathcal{G}$. Set $\mathcal{D}_{n+1}=\mathcal{D}_{n+1}^{r}=\left(s_{i}^{n+1}\right)_{i \in \mathbb{N}}$. Then, according to (ii), $\left[\mathbf{s} * \mathbf{t}_{i}, \mathcal{D}_{n+1}\right] \in \mathcal{G}$ for every $1 \leq i \leq r$.

Set $\mathcal{D}_{0}=\left(s_{1}, \ldots, s_{k}, s_{1}^{1}, s_{2}^{2}, \ldots\right) \in \mathcal{B}^{\omega}(\mathbb{N})$. Then $\mathcal{D}_{0} \in[\mathbf{s}, \mathcal{D}]$. Let $\mathbf{t} \in \mathcal{B}^{<\omega}\left(\mathcal{D}_{0}-\mathbf{s}\right)$ with $\mathbf{t} \neq \emptyset$. If $n_{0}=\min \left\{n \in \mathbb{N}: \sigma(\mathbf{t}) \subseteq F U\left(\left\{s_{1}^{1}, \ldots, s_{n}^{n}\right\}\right)\right\}$, then $\left[\mathbf{s} * \mathbf{t}, \mathcal{D}_{n_{0}+1}\right] \in \mathcal{G}$. According to assumption (ii), $\left[\mathbf{s} * \mathbf{t}, \overline{\mathcal{D}}_{0}-s_{n_{0}}^{n_{0}}\right] \in \mathcal{G}$. Hence, $\left[\mathbf{s} * \mathbf{t}, \mathcal{D}_{0}\right]=[\mathbf{s} * \mathbf{t}$, $\left.\mathcal{D}_{0}-s_{n_{0}}^{n_{0}}\right] \in \mathcal{G}$. If $\mathbf{t}=\emptyset$, then $\left[\mathbf{s}, \mathcal{D}_{1}\right] \in \mathcal{G}$, hence $\left[\mathbf{s}, \mathcal{D}_{0}\right] \in \mathcal{G}$.

Proof of Theorem 5.2 , Let $\mathcal{U} \in \mathcal{B}^{\omega}(\mathbb{N}), \mathbf{s} \in \mathcal{B}^{<\omega}(\mathbb{N})$ and $\mathcal{D} \in \mathcal{B}^{\omega}(\mathbb{N})$. Set

$$
\begin{aligned}
& \mathcal{G}_{\mathcal{U}}=\left\{[\mathbf{s}, \mathcal{D}]:(\mathbf{s}, \mathcal{D}) \in \mathcal{B}^{<\omega}(\mathbb{N}) \times \mathcal{B}^{\omega}(\mathbb{N})\right. \text { and } \\
&\text { either } \left.[\mathbf{s}, \mathcal{D}] \cap \mathcal{U}=\emptyset \text { or } \quad\left[\mathbf{s}, \mathcal{D}_{1}\right] \cap \mathcal{U} \neq \emptyset \text { for every } \mathcal{D}_{1}<\mathcal{D}\right\} .
\end{aligned}
$$

It is easy to check that $\mathcal{G}_{\mathcal{U}}$ satisfies assumptions (i) and (ii) of Lemma 5.3. hence, there exists $\mathcal{D}_{0} \in[\mathbf{s}, \mathcal{D}]$ such that $\left[\mathbf{s} * \mathbf{t}, \mathcal{D}_{0}\right] \in \mathcal{G}_{\mathcal{U}}$ for every $\mathbf{t} \in \mathcal{B}^{<\omega}\left(\mathcal{D}_{0}-\mathbf{s}\right)$. Set

$$
\mathcal{F}=\left\{\mathbf{t} \in \mathcal{B}^{<\omega}\left(\mathcal{D}_{0}-s\right):\left[\mathbf{s} * \mathbf{t}, \mathcal{D}_{1}\right] \cap \mathcal{U} \neq \emptyset \text { for every } \mathcal{D}_{1}<\mathcal{D}_{0}\right\} .
$$

The family $\mathcal{F}$ is a tree. Indeed, let $\mathbf{t} \in \mathcal{F}$ and $\mathbf{t}_{1} \propto \mathbf{t}$. Then $\left[\mathbf{s} * \mathbf{t}_{1}, \mathcal{D}_{0}\right] \in \mathcal{G}_{\mathcal{U}}$, since $\mathbf{t}_{1} \in \mathcal{B}^{<\omega}\left(\mathcal{D}_{0}-\mathbf{s}\right)$. So either $\left[\mathbf{s} * \mathbf{t}_{1}, \mathcal{D}_{0}\right] \cap \mathcal{U}=\emptyset$ which is impossible, since $\left[\mathbf{s} * \mathbf{t}, \mathcal{D}_{0}\right] \cap \mathcal{U} \neq \emptyset$, or $\left[\mathbf{s} * \mathbf{t}_{1}, \mathcal{D}_{1}\right] \cap \mathcal{U} \neq \emptyset$ for every $\mathcal{D}_{1}<\mathcal{D}_{0}$. Hence $\mathbf{t}_{1} \in \mathcal{F}$.

We use Theorem 4.6 for $\mathcal{F}$ and $\mathcal{D}_{0}-s$. We have the following cases:

[Case 1] There exists $\mathcal{D}_{1}<\mathcal{D}_{0}-s<\mathcal{D}$ such that $\mathcal{B}^{<\omega}\left(\mathcal{D}_{1}\right) \subseteq \mathcal{F}$. This gives that $\left[\mathbf{s} * \mathbf{t}, \mathcal{D}_{2}\right] \cap \mathcal{U} \neq \emptyset$ for every $\mathbf{t} \in \mathcal{B}^{<\omega}\left(\mathcal{D}_{1}\right)$ and $\mathcal{D}_{2}<\mathcal{D}_{1}$, which implies that $\left[\mathbf{s}, \mathcal{D}_{1}\right] \subseteq \hat{\mathcal{U}}$

[Case 2] There exists a countable ordinal $\xi_{0}=\zeta_{\mathcal{D}}^{\mathcal{F}}=\zeta_{(\mathbf{s}, \mathcal{D})}^{\mathcal{U}}$ such that for every $\xi>\xi_{0}$ there exists $\mathcal{D}_{1}<\mathcal{D}_{0}-\mathbf{s}<\mathcal{D}$ with $\mathcal{B}^{\xi} \cap \mathcal{B}^{<\omega}\left(\mathcal{D}_{1}\right) \subseteq \mathcal{B}^{<\omega}(\mathbb{N}) \backslash \mathcal{F}$. Then $\left[\mathbf{s} * \mathbf{t}, \mathcal{D}_{1}\right] \subseteq \mathcal{B}^{\omega}(\mathbb{N}) \backslash \mathcal{U}$ for every $\mathbf{t} \in \mathcal{B}^{\xi} \cap \mathcal{B}^{<\omega}\left(\mathcal{D}_{1}\right)$.

Applying Theorem 5.2 to partitions $\mathcal{U}$ that are closed (the pointwise closed families are included in this class) or meager in the topology $\mathfrak{T}_{E}$, we consider the following consequences.

Corollary 5.4. Let $\mathcal{U}$ be a subset of $\mathcal{B}^{\omega}(\mathbb{N})$ pointwise closed (or, even $\mathcal{U}$ closed in the topology $\left.\mathfrak{T}_{E}\right)$, let $\mathbf{s} \in \mathcal{B}^{<\omega}(\mathbb{N})$ and let $\mathcal{D}$ be an infinite-ordered disjoint collection. Then

either there exists $\mathcal{D}_{1}<\mathcal{D}$ such that $\left[\mathbf{s}, \mathcal{D}_{1}\right] \subseteq \mathcal{U}$,

or there exists a countable ordinal $\xi_{0}=\xi_{(\mathbf{s}, \mathcal{D})}^{\mathcal{U}}$, such that for every $\xi>\xi_{0}$ there exists $\mathcal{D}_{1}<\mathcal{D}-\mathbf{s}$ such that $\left[\mathbf{s} * \mathbf{t}, \mathcal{D}_{1}\right] \subseteq \mathcal{B}^{\omega}(\mathbb{N}) \backslash \mathcal{U}$ for every $\mathbf{t} \in$ $\mathcal{B}^{\xi} \cap \mathcal{B}^{<\omega}\left(\mathcal{D}_{1}\right)$.

Proof. It follows from Theorem 5.2

Corollary 5.5. Let $\mathcal{U}$ be a subset of $\mathcal{B}^{\omega}(\mathbb{N})$ meager in the topology $\mathfrak{T}_{E}$, let $\mathbf{s} \in$ $\mathcal{B}^{<\omega}(\mathbb{N})$ and let $\mathcal{D} \in \mathcal{B}^{\omega}(\mathbb{N})$. Then, there exists a countable ordinal $\xi_{0}$ such that for every $\xi>\xi_{0}$ there exists $\mathcal{D}_{1}<\mathcal{D}-\mathbf{s}$ such that $\left[\mathbf{s} * \mathbf{t}, \mathcal{D}_{1}\right] \subseteq \mathcal{B}^{\omega}(\mathbb{N}) \backslash \mathcal{U}$ for every $\mathbf{t} \in \mathcal{B}^{\xi} \cap \mathcal{B}^{<\omega}\left(\mathcal{D}_{1}\right)$.

Proof. We use Theorem 5.2 for $\mathcal{U}$. We will prove that the first alternative is impossible. Indeed, let $\mathcal{D}_{1}<\mathcal{D}$ such that $\left[\mathbf{s}, \mathcal{D}_{1}\right] \subseteq \hat{\mathcal{U}}$. We denote by $|\mathbf{t}|$ the cardinality 
of $\sigma(\mathbf{t})$ for a $\mathbf{t} \in \mathcal{B}^{<\omega}(\mathbb{N})$. If $\mathcal{U}=\bigcup_{n \in \mathbb{N}} \mathcal{U}_{n}$ with $\left(\hat{\mathcal{U}}_{n}\right)^{\diamond}=\emptyset$ for every $n \in \mathbb{N}$, then we set

$$
\begin{aligned}
\mathcal{G}=\left\{\left[\mathbf{t}, \mathcal{D}_{0}\right]: \mathbf{t} \in \mathcal{B}^{<\omega}(\mathbb{N}), \mathcal{D}_{0} \in \mathcal{B}^{\omega}(\mathbb{N})\right. \text { and } \\
\left.\quad\left[\mathbf{t}, \mathcal{D}_{0}\right] \cap \mathcal{U}_{k}=\emptyset \text { for every } k \in \mathbb{N} \text { with } k \leq|\mathbf{t}|\right\} .
\end{aligned}
$$

The family $\mathcal{G}$ satisfies conditions (i) and (ii) of Lemma 5.3 . Indeed, according to Theorem 5.2, for every $\mathbf{t} \in \mathcal{B}^{<\omega}(\mathbb{N}), \mathcal{D} \in \mathcal{B}^{\omega}(\mathbb{N})$ and $k \in \mathbb{N}$ there exists $\mathcal{D}_{0}^{\prime}<\mathcal{D}$ such that $\left[\mathbf{t}, \mathcal{D}_{0}^{\prime}\right] \cap \mathcal{U}_{k}=\emptyset$, as it is impossible that $\left[\mathbf{t}, \mathcal{D}_{0}^{\prime}\right] \subseteq \hat{\mathcal{U}}_{k}$. Thus $\mathcal{G}$ satisfies (i) and obviously satisfies (ii). Hence, according to Lemma 5.3 , there exists $\mathcal{D}_{2} \in\left[\mathbf{s}, \mathcal{D}_{1}\right]$ such that $\left[\mathbf{s} * \mathbf{t}, \mathcal{D}_{2}\right] \in \mathcal{G}$ for every $\mathbf{t} \in \mathcal{B}^{<\omega}\left(\mathcal{D}_{2}-\mathbf{s}\right)$. Then, $\left[\mathbf{s}, \mathcal{D}_{2}\right] \cap \mathcal{U}=\emptyset$. Indeed, let $\mathcal{D}_{3} \in\left[\mathbf{s}, \mathcal{D}_{2}\right] \cap \mathcal{U}$. Then, $\mathcal{D}_{3} \in\left[\mathbf{s}, \mathcal{D}_{2}\right] \cap \mathcal{U}_{k}$ for some $k \in \mathbb{N}$. Hence, there exists $\mathbf{t} \in \mathcal{B}^{<\omega}\left(\mathcal{D}_{2}-\mathbf{s}\right)$ with $\mathbf{s} * \mathbf{t} \propto \mathcal{D}_{3}, k \leq|\mathbf{s} * \mathbf{t}|$ and $\left[\mathbf{s} * \mathbf{t}, \mathcal{D}_{2}\right] \cap \mathcal{U}_{k} \neq \emptyset$. Then, $\left[\mathbf{s} * \mathbf{t}, \mathcal{D}_{2}\right] \notin \mathcal{G}$. A contradiction, since $\mathbf{t} \in \mathcal{B}^{<\omega}\left(\mathcal{D}_{2}-\mathbf{s}\right)$. Thus, $\left[\mathbf{s}, \mathcal{D}_{2}\right] \cap \mathcal{U}=\emptyset$. This implies that $\mathcal{D}_{2} \notin \hat{\mathcal{U}}$, which is a contradiction, since $\mathcal{D}_{2} \in\left[\mathbf{s}, \mathcal{D}_{1}\right] \subseteq \hat{\mathcal{U}}$. Hence, the first alternative of Theorem 5.2 for the partition $\mathcal{U}$ is impossible, so the second alternative holds for $\mathcal{U}$.

We recall the definition of the completely Ramsey families of infinite-ordered disjoint collection given in $[\mathrm{M}]$.

Definition 5.6. A family $\mathcal{U} \subseteq \mathcal{B}^{\omega}(\mathbb{N})$ of infinite-ordered disjoint collections is called completely Ramsey if for every $\mathbf{s} \in \mathcal{B}^{<\omega}(\mathbb{N})$ and every $\mathcal{D} \in \mathcal{B}^{\omega}(\mathbb{N})$ there exists $\mathcal{D}_{1}<\mathcal{D}$ such that

$$
\text { either }\left[\mathbf{s}, \mathcal{D}_{1}\right] \subseteq \mathcal{U} \quad \text { or } \quad\left[\mathbf{s}, \mathcal{D}_{1}\right] \subseteq \mathcal{B}^{\omega}(\mathbb{N}) \backslash \mathcal{U}
$$

A further consequence of Theorem 5.2 is the following corollary which gives the characterization of completely Ramsey families (also proved in [M] and independently in [T1]).

Corollary 5.7. A family $\mathcal{U} \subseteq \mathcal{B}^{\omega}(\mathbb{N})$ is completely Ramsey if and only if $\mathcal{U}$ has the Baire property in the topology $\mathfrak{T}_{E}$.

Proof. Let $\mathcal{U} \subseteq \mathcal{B}^{\omega}(\mathbb{N})$ have the Baire property in the topology $\mathfrak{T}_{E}$. Then $\mathcal{U}=$ $\mathcal{B} \triangle \mathcal{C}=\left(\mathcal{B} \cup \mathcal{C}^{c}\right) \cup\left(\mathcal{C} \cap \mathcal{B}^{c}\right)$, where $\mathcal{B} \subseteq \mathcal{B}^{\omega}(\mathbb{N})$ is $\mathfrak{T}_{E^{-c l o s e d}}$ and $\mathcal{C} \subseteq \mathcal{B}^{\omega}(\mathbb{N})$ is $\mathfrak{T}_{E^{-}}$ meager $\left(\mathcal{C}^{c}=\mathcal{B}^{\omega}(\mathbb{N}) \backslash \mathcal{C}\right)$. According to Corollaries 5.4 and 5.5, for every $\mathbf{s} \in \mathcal{B}^{<\omega}(\mathbb{N})$ and $\mathcal{D} \in \mathcal{B}^{\omega}(\mathbb{N})$, there exists $\mathcal{D}_{1}<\mathcal{D}$ such that $\left[\mathbf{s}, \mathcal{D}_{1}\right] \subseteq \mathcal{C}^{c}$ and consequently there exists $\mathcal{D}_{2}<\mathcal{D}_{1}$ such that

either $\left[\mathbf{s}, \mathcal{D}_{2}\right] \subseteq \mathcal{B} \cap\left[\mathbf{s}, \mathcal{D}_{1}\right] \subseteq \mathcal{B} \cap \mathcal{C}^{c} \subseteq \mathcal{U}$

or $\left[\mathbf{s}, \mathcal{D}_{2}\right] \subseteq \mathcal{B}^{c} \cap\left[\mathbf{s}, \mathcal{D}_{1}\right] \subseteq \overline{\mathcal{B}}^{c} \cap \mathcal{C}^{c} \subseteq \mathcal{U}^{c}$

Hence, $\mathcal{U}$ is completely Ramsey.

On the other hand, if $\mathcal{U}$ is completely Ramsey, then $\mathcal{U}=\mathcal{U}^{\diamond} \cup\left(\mathcal{U} \backslash \mathcal{U}^{\diamond}\right)$ and $\mathcal{U} \backslash \mathcal{U}^{\diamond}$ is a meager set in $\mathfrak{T}_{E}$. Hence $\mathcal{U}$ has the Baire property in the topology $\mathfrak{T}_{E}$.

Remark 5.8. (i) Every subset $\mathcal{U}$ of $\mathcal{B}^{\omega}(\mathbb{N})$ which is a Borel set in the topology of pointwise convergence is completely Ramsey, since it has the Baire property in the topology $\mathfrak{T}_{E}$.

(ii) Every subset of $\mathcal{B}^{\omega}(\mathbb{N})$ which is an analytic set in the topology of pointwise convergence is completely Ramsey, since every analytic set (in this topology) has the Baire property. 


\section{ACKnowledgments}

We wish to express our indebtedness to the anonymous referee for detailed, indepth suggestions that led to substantial improvements of the paper. Thanks are due to Ted Odell and Haskell Rosenthal for helpful comments and discussions on the content of this paper and also to the Department of Mathematics of the University

of Texas at Austin for the hospitality extended during our visit during the spring semester of 2004.

\section{REFERENCES}

[AA] D. Alspach and S. Argyros, Complexity of weakly null sequences, Dissertations Math. 321 (1992), 1-44. MR1191024 (93j:46014)

[AO] D. Alspach and E. Odell, Averaging weakly null sequences, Lecture Notes in Math. 1332, Springer, Berlin, 1988, pp. 126-144. MR0967092 (89j:46014)

[AGR] S. Argyros, G. Godefroy and H. Rosenthal, Descriptive set theory and Banach spaces, Handbook of the geometry of Banach spaces 2, North-Holland, Amsterdam (2003), 10071069. MR 1999190 (2004g:46002)

[B] J. Baumgartner, A short proof of Hindman's theorem, J. Combinatorial Theory, Ser. A, 17 (1974), 384-386. MR0354394 (50:6873)

[E] E. Ellentuck, A new proof that analytic sets are Ramsey, J. Symb. Logic 39 (1974), 163164. MR0349393 (50:1887)

[F1] V. Farmaki, Classifications of Baire-1 functions and $c_{0}$-spreading models, Trans. Amer. Math. Soc. 345 (2), (1994), 819-831. MR.1262339 (96c:46017)

[F2] V. Farmaki, Ramsey dichotomies with ordinal index, arXiv: math. LO/9804063 v1, (1998), electronic prepublication.

[F3] V. Farmaki, Ramsey and Nash-Williams combinatorics via Schreier families, arXiv: math. FA/0404014 v.1, (2004), electronic prepublication.

[F4] V. Farmaki, The uniform convergence ordinal index and the $l^{1}$-behavior of a sequence of functions, Positivity 8 (1), (2004), 49-74. MR2053575 (2005e:46029)

[G] W. T. Gowers, An infinite Ramsey theorem and some Banach space dichotomies, Annals of Mathematics 156 (2002), 797-833. MR.1954235(2005a:46032)

[GRS] R. Graham, B. Rothschild and J. Spencer, Ramsey Theory, Wiley, New York, 1990. MR 1044995 (90m:05003)

$[\mathrm{H}] \quad$ N. Hindman, Finite sums from sequences within cells of a partition of $\mathbb{N}$, J. Combinatorial Theory, Ser. A 17 (1974), 1-11. MR0349574 (50:2067)

[KS] J. Ketonen and R. Solovay, Rapidly growing Ramsey functions, Ann. of Math. 113 (1981), 267-314. MR0607894 (84c:03100)

[K] K. Kuratowski, Topology, Volume I, Academic Press (1966). MR 0217751 (36:840)

[KM] K. Kuratowski and A. Mostowski, Set Theory, North-Holland, Amsterdam, 1968.

[L] A. Levy, Basic Set Theory, Springer-Verlag, 1979. MR0533962 (80k:04001)

[M] K. Milliken, Ramsey's theorem with sums or unions, J. Combinatorial Theory, Ser. A 18 (1975), 276-290. MR0373906 (51:10106)

[NW] C. St. J. A. Nash-Williams, On well quasiordering transfinite sequences, Proc. Camb. Phil. Soc. 61 (1965), 33-39. MR0173640 (30:3850)

[O] E. Odell, On subspaces, asymptotic structure, and distortion of Banach spaces; connections with logic, Analysis and Logic, London Math. Soc. Lecture Note Ser. 262, Cambridge Univ. Press (2002), 189-267. MR.1967836 (2004b:46016)

$[\mathrm{PH}]$ J. Paris and L. Harrington, A mathematical incompleteness in Peano arithmetic, Handbook of Mathematical Logic, North-Holland, Amsterdam (1977), 1133-1142. MR0457132 $(56: 15351)$

[R] F. P. Ramsey, On a problem of formal logic, Proc. London Math. Soc. 30 (2), (1929), 264-286.

[S] J. Schreier, Ein Gegenbeispiel zur Theorie der schwachen Konvergenz, Studia Math. 2 (1930), 58-62.

[T1] A. Taylor, Some results in partition theory, Ph.D. dissertation, Dartmouth College, 1975. 
[T2] A. Taylor, A canonical partition relation for finite subsets of $\omega$, J. Combinatorial Theory, Ser. A 21 (1976), 137-146. MR0424571 (54:12530)

[TJ] N. Tomczak-Jaegermann, Banach spaces of type p have arbitrarily distortable subspaces, Geom. As. Funct. Anal. 6 (1996), 1074-1082. MR.1421875 (98g:46020)

Department of Mathematics, Athens University, Athens 157 84, Greece

E-mail address: vfarmaki@math.uoa.gr

Department of Mathematics, Athens University, Athens 157 84, Greece

E-mail address: snegrep@math.uoa.gr 